\title{
Schur sector of Argyres-Douglas theory and $W$-algebra
}

\author{
Dan Xie ${ }^{a, b}$ Wenbin Yan $^{a}$ \\ ${ }^{a}$ Yau Mathematics Science center, Tsinghua University, Beijing, 10084, China \\ ${ }^{b}$ Department of Mathematics, Tsinghua University, Beijing, 10084, China
}

\begin{abstract}
We study the Schur index, the Zhu's $C_{2}$ algebra, and the Macdonald index of a four dimensional $\mathcal{N}=2$ Argyres-Douglas (AD) theories from the structure of the associated two dimensional $W$-algebra. The Schur index is derived from the vacuum character of the corresponding $W$-algebra and can be rewritten in a very simple form, which can be easily used to verify properties like level-rank dualities, collapsing levels, and S-duality conjectures. The Zhu's $C_{2}$ algebra can be regarded as a ring associated with the Schur sector, and a surprising connection between certain Zhu's $C_{2}$ algebra and the Jacobi algebra of a hypersurface singularity is discovered. Finally, the Macdonald index is computed from the Kazhdan filtration of the $W$-algebra.
\end{abstract}




\section{Contents}

1 Introduction 1

2 Schur sector and VOA $\quad 4$

2.1 Quasi-lisse VOA 4

$2.24 \mathrm{~d} / 2 \mathrm{~d}$ correspondence $\quad 7$

2.3 Comments on constraints of 2d VOAs corresponding to 4d SCFTs 7

3 Argyres-Douglas theories and $W$-algebras $\quad 8$

3.1 AD theories correspond to $W^{k^{\prime}}(\mathfrak{g}, f)$ algebras 8

4 The character of $W$-algebra and the Schur index 10

$\begin{array}{ll}4.1 \text { The } W \text {-algebra from the qDS reduction } & 10\end{array}$

$\begin{array}{ll}4.2 \text { Character of } W \text {-algebra modules } & 12\end{array}$

$\begin{array}{ll}4.3 \text { The simplified form } & 13\end{array}$

$\begin{array}{ll}\text { 4.3.1 Applications } & 14\end{array}$

$\begin{array}{lll}4.4 & \text { Explicit indices of }\left(A_{1}, G\right) \text { theories } & 18\end{array}$

$\begin{array}{lll}4.5 & \tau \rightarrow 0 \text { limit and } a_{4 d}-c_{4 d} & 19\end{array}$

5 Zhu's $C_{2}$ algebra and the ring of the Schur sector $\quad 21$

$\begin{array}{lll}5.1 \text { Zhu's } C_{2} \text { algebra and Jacobi algebra } & 21\end{array}$

5.2 Singular vector and general proposal for Zhu's $C_{2}$ algebra 23

$\begin{array}{ll}5.3 \text { A general proposal for Zhu's } C_{2} \text { algebra } & 25\end{array}$

6 Kazhdan filtration and Macdonald index 26

$\begin{array}{llr}7 & \text { Conclusion } & 28\end{array}$

A Notation of special functions $\quad 30$

\section{Introduction}

It is important to understand moduli spaces of vacua of four dimensional $\mathcal{N}=2$ superconformal field theories (SCFTs). An $\mathcal{N}=2$ SCFT could have a Coulomb branch and a Higgs branch. The low energy effective theory on the Coulomb branch is solved by finding a Seiberg-Witten geometry. Almost every nontrivial four dimensional $\mathcal{N}=2$ SCFT has

a Coulomb branch, which is parameterized by expectation values of half-BPS operators 
$\mathcal{E}_{r,(0,0)}{ }^{1}$. These operators form a ring which is freely generated for almost all the theories we know ${ }^{2}$. The important question is to determine the rational number $r$ of each Coulomb branch operator $\mathcal{E}_{r,(0,0)}$. In practice, one can often easily determine them using the Seiberg-Witten (SW) geometry.

It is also possible for a four dimensional $\mathcal{N}=2$ SCFT to have a Higgs branch, which is parameterized by expectation values of half-BPS operators $\hat{\mathcal{B}}_{R}$. These operators form a nontrivial ring called the Higgs branch chiral ring. Unlike the common appearance of the Coulomb branch, not all $\mathcal{N}=2$ SCFT has a Higgs branch and in fact there does exist a large class of $\mathcal{N}=2$ SCFTs which do not have a Higgs branch.

Given the asymmetry between the Higgs branch and the Coulomb branch, one might wonder whether a protected sector could exist for all non-trivial $\mathcal{N}=2$ SCFT and contains the Higgs branch when the theory has one. Such sector indeed exists and is called the Schur sector $[3,4]$, which contains Higgs branch operators $\hat{\mathcal{B}}_{R}$ and operators $\hat{\mathcal{C}}_{R,\left(j_{1}, j_{2}\right)}$. It is in general quite difficult to determine this sector as there is no powerful tool as the SW geometry of the Coulomb branch.

The understanding of the Schur sector becomes possible because of the following $4 \mathrm{~d} / 2 \mathrm{~d}$ correspondence found in [5] (see [6-55] for further developments). There is a map between the Schur sector of a $4 \mathrm{~d} \mathcal{N}=2 \mathrm{SCFT}$ and a $2 \mathrm{~d}$ vertex operator algebra (VOA). Once the $2 \mathrm{~d}$ VOA for a $4 \mathrm{~d} \mathcal{N}=2 \mathrm{SCFT}$ is identified, one can learn a lot about the Schur sector of the $4 \mathrm{~d}$ theory from known properties of $2 \mathrm{~d}$ VOA.

For a large class of $4 \mathrm{~d} \mathcal{N}=2$ Argyres-Douglas type SCFTs engineered from $6 \mathrm{~d}(2,0)$ theories, we have identified their associated 2d VOAs as $W$-algebras $W^{k^{\prime}}(\mathfrak{g}, f)[17,29$, 56] shown in figure 1 . Such $W$-algebra is derived from the quantum Drinfeld-Sokolov (qDS) reduction of an affine Kac-Moody (AKM) algebra $V^{k^{\prime}}(\mathfrak{g})$ with $k^{\prime}$ being a boundary admissible level $k^{\prime}=-h^{\vee}+\frac{h^{\vee}}{h^{\vee}+k}{ }^{3}$, and $f$ is a nilpotent element of Lie algebra $\mathfrak{g}$.

Such $W$-algebra has been studied in physics and mathematics literature extensively[5961]. The purpose of this paper is to extract important information of the Schur sector of the $4 \mathrm{~d}$ theory from the knowledge of the $2 \mathrm{~d}$ VOA. We obtain three main results:

1. The Schur index can be computed from the vacuum character of the $W$-algebra $W^{k^{\prime}}(\mathfrak{g}, f)$, and the character has a very elegant product formula for the boundary admissible level [62]. We discovered that the index can be put as a very simple form

$$
\mathcal{I}_{W^{k^{\prime}}(\mathfrak{g}, f)}(q, z)=P E\left[\frac{\sum_{j} q^{1+j} \chi_{R_{j}}(z)-q^{h^{\vee}+k} \sum_{j} q^{-j} \chi_{R_{j}}(z)}{(1-q)\left(1-q^{h^{\vee}+k}\right)}\right] .
$$

Here for a nilpotent element $f$, one has an associated $\mathfrak{s l}_{2}$ triple and the associated Lie group $G$ of $\mathfrak{g}$ has a subgroup $S U(2) \times G_{F}$ with $G_{F}$ being the flavor symmetry group

\footnotetext{
${ }^{1} \mathcal{N}=2$ SCFT has a bosonic symmetry group $S O(2,4) \times S U(2)_{R} \times U(1)_{R}$, and the highest weight representation is labeled as $\left|\Delta, R, r, j_{1}, j_{2}\right\rangle$, here $\Delta$ is the scaling dimension, $R$ labels the $S U(2)_{R}$ representation, $r$ is $U(1)_{R}$ charge, and $j_{1}, j_{2}$ are left and right spins. Short supermultiplets are classified in [1], and there are three types of half BPS operators which are important to us: a): $\mathcal{E}_{r,(0,0)}$ with $\Delta=r$ and $\left.R=0 ; \mathrm{b}\right): \hat{\mathcal{B}}_{R}$ with $\left.\Delta=2 R, r=j_{1}=j_{2}=0 ; \mathrm{c}\right): \hat{\mathcal{C}}_{R,\left(j_{1}, j_{2}\right)}$ with $\Delta=2+2 R+j_{1}+j_{2}$ and $r=j_{2}-j_{1}$.

${ }^{2}$ See [2] for the discussion on the possibility of nontrivial Coulomb branch chiral ring.

${ }^{3}$ Here $\mathfrak{g}$ is a simple Lie algebra, $h^{\vee}$ is its dual Coxeter number and $\mathrm{k}$ is an integer with following constraints: a) $h^{\vee}+k \geq 2$; b) $k$ and $h^{\vee}$ coprime; c) and $k \neq 2 n$ for $\mathfrak{g}=B_{N}, C_{N}, F_{4}$, and $k \neq 3 n$ for $\mathfrak{g}=G_{2}$.
} 


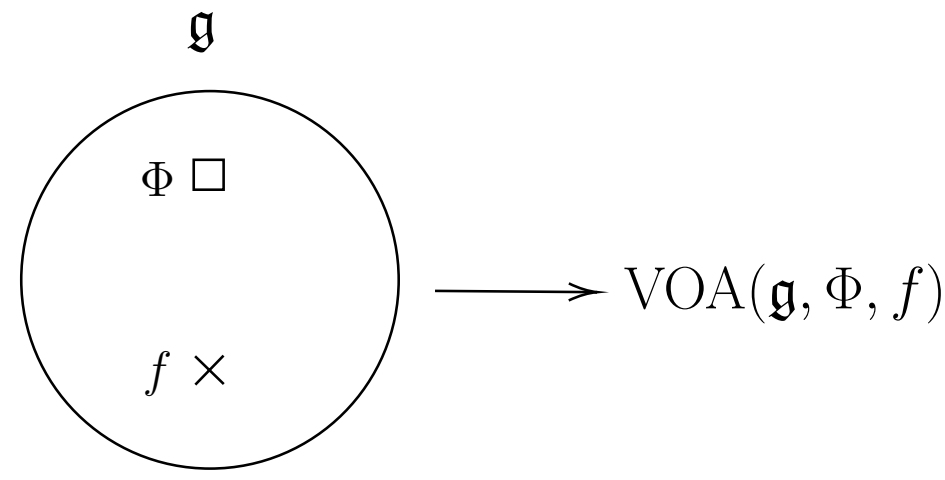

Figure 1: A mapping of a $6 \mathrm{~d}(2,0)$ configuration to a $2 \mathrm{~d}$ VOA, here $\mathfrak{g}$ is a simple Lie algebra, $\Phi$ is an irregular singularity, and $f$ represents a regular singularity. If $\Phi$ is of principle nilpotent type with type $h^{\vee}$ and an integer label $k[39,57,58]$, the VOA is just $W$-algebra $W^{k^{\prime}}(\mathfrak{g}, f)$ with $k^{\prime}=-h^{\vee}+\frac{h^{\vee}}{h^{\vee}+k}$.

corresponding to $f$. The adjoint representation of $\mathfrak{g}$ decomposes as $a d j_{\mathfrak{g}} \rightarrow \oplus_{j} V_{j} \otimes R_{j}$ under the subgroup $S U(2) \times G_{F}$, here $j$ is the spin $j$ representation of $S U(2)$ subgroup, and $R_{j}$ is the representation under the flavor group $G_{F} \cdot \chi_{R_{j}}(z)$ is the character of representation $R_{j}$ of flavor group $G_{F}$. This formula is a generalization of $\mathfrak{g}=A D E$ case considered in [29] to the arbitrary simple Lie algebra. With this simple form, we can check many highly nontrivial properties of $4 \mathrm{~d}$ and $2 \mathrm{~d}$ theories, i.e. levelrank dualities and collapsing levels of $2 \mathrm{~d}$ VOA, and more interestingly the S-duality conjecture proposed in [63, 64].

2. For a VOA, one can define a commutative and associative algebra called the Zhu's $C_{2}$ algebra [65]. The reduced ring from the $C_{2}$ algebra is identified with the Higgs branch chiral ring $[29,31,66]$. Our new point of view in this paper is that the Zhu's $C_{2}$ algebra is more important than its reduced version, and it can be thought as the associated ring of the Schur sector. For our cases, the Zhu's $C_{2}$ algebra has a simple form and we gave a general proposal for its structure. If our $4 \mathrm{~d}$ theory has no flavor symmetry, we conjecture that the $C_{2}$ algebra is actually isomorphic to a Jacobi algebra associated with a quasi-homogenous hypersurface singularity.

3. To compute the Macdonald index of a given $4 \mathrm{~d}$ theory, one need to introduce another grading or filtration in the corresponding VOA. For our $W$-algebra, there is a natural filtration called the Kazhdan filtration [67] which we use to define the Macdonald index of the $4 \mathrm{~d}$ theory. This filtration agrees with the filtration of theories considered in [22], and gives a natural generalization to general models considered in this paper.

This paper is organized as follows: section 2 reviews the basic correspondence between the Schur sector of $4 \mathrm{~d}$ theory and $2 \mathrm{~d}$ VOA. Section 3 reviews known results between $4 \mathrm{~d}$ Argyres-Douglas theories engineered from $6 \mathrm{~d}(2,0)$ theories and their associated $2 \mathrm{~d} W$ algebras. Section 4 studies the Schur index from the vacuum character of the $W$-algebra. Section 5 studies the Zhu's $C_{2}$ algebra which might be thought of as a ring associated with 
the Schur sector. Section 6 introduces the Kazhdan filtration of our $W$-algebra and it is used to define the Macdonald index. Finally a conclusion is given in section 7 .

\section{Schur sector and VOA}

The representation theory of a $4 \mathrm{~d} \mathcal{N}=2$ SCFT was studied in [1]. $\mathcal{N}=2$ SCFT has a bosonic symmetry group $S O(2,4) \times S U(2)_{R} \times U(1)_{R}$, and the highest weight representation is labeled as $\left|\Delta, R, r, j_{1}, j_{2}\right\rangle$, here $\Delta$ is the scaling dimension, $R$ labels the $S U(2)_{R}$ representation, $r$ is $U(1)_{R}$ charge, and $j_{1}, j_{2}$ are left and right spins. Short supermultiplets are classified in [1], and there are three types of half BPS operators which are important to us: a): $\mathcal{E}_{r,(0,0)}$ with $\Delta=r$ and $\left.R=0 ; \mathrm{b}\right): \hat{\mathcal{B}}_{R}$ with $\left.\Delta=2 R, r=j_{1}=j_{2}=0 ; \mathrm{c}\right): \hat{\mathcal{C}}_{R,\left(j_{1}, j_{2}\right)}$ with $\Delta=2+2 R+j_{1}+j_{2}$ and $r=j_{2}-j_{1}$. We are interested in so-called Schur sector which contains operators satisfying the following condition

$$
\begin{aligned}
& \frac{1}{2}\left(\Delta-\left(j_{1}+j_{2}\right)\right)-R=0, \\
& r+j_{1}-j_{2}=0 .
\end{aligned}
$$

The Schur operators are contained in supermultiplets $\hat{\mathcal{C}}_{R,\left(j_{1}, j_{2}\right)}, \hat{\mathcal{B}}_{R}, \mathcal{D}_{0\left(0, j_{2}\right)}$ and $\overline{\mathcal{D}}_{0\left(j_{1}, 0\right)}$. $\mathcal{D}_{0\left(0, j_{2}\right)}$ and $\overline{\mathcal{D}}_{0\left(j_{1}, 0\right)}$ multiplets will not appear in theories considered here [68]. $\hat{\mathcal{C}}_{0,(0,0)}$ is the supercurrent multiplet and $\hat{\mathcal{B}}_{R}$ multiplets contain Higgs branch operators. Notice that for $\hat{\mathcal{C}}$ type supermultiplet, the Schur operator is not the bottom component.

The Macdonald index and the Schur index $[3,4]$ are non-zero in the Schur sector only. The Macdonald index of a $\hat{\mathcal{C}}_{R,\left(j_{1}, j_{2}\right)}$ multiplet is ${ }^{4}$

$$
\mathcal{I}_{\hat{\mathcal{C}}_{R,\left(j_{1}, j_{2}\right)}^{M}}(q, T)=\frac{q^{2+R+j_{1}+j_{2}} T^{1+R+j_{2}-j_{1}}}{1-q},
$$

where $\frac{1}{1-q}$ represents the contribution from derivatives. The Schur index of the same multiplet is given by setting $T=1$ in above formula

$$
\mathcal{I}_{\hat{\mathcal{C}}_{R,\left(j_{1}, j_{2}\right)}^{\text {Schur }}}(q)=\frac{q^{2+R+j_{1}+j_{2}}}{1-q} .
$$

Moreover, if the theory has a flavor symmetry, one may also add flavor fugacities in both indices, which keep track of the action of the flavor group. Such fugacities are crucial when considering modular properties of indices. Another important property is that Higgs branch operators $\hat{\mathcal{B}}_{R}$ form a ring and in most cases there is also a Hyperkhaler metric associated with this ring.

\subsection{Quasi-lisse VOA}

VOA arises as the chiral part of a two dimensional conformal field theory. Here we review the mathematical definition of a VOA. A vertex algebra is a vector space $V$ with following properties ( $V$ can be thought of as the vacuum module of the chiral part of $2 \mathrm{~d}$ CFT) [69]:

\footnotetext{
${ }^{4}$ We use the notation $\hat{\mathcal{B}}_{R+1}=\hat{\mathcal{C}}_{R,\left(-\frac{1}{2},-\frac{1}{2}\right)}$.
} 
- A vacuum vector $|0\rangle$.

- A linear map

$$
Y: V \rightarrow \mathcal{F}(V), \quad a \rightarrow Y(a, z)=\sum_{n} a_{n} z^{-n-1}=a(z),
$$

where $a_{n} \in \operatorname{End}(V)$. This is just the state-operator correspondence ${ }^{5}$. Given a field $a(z)$, one can recover the corresponding state $|a(z)\rangle=\lim _{z \rightarrow 0} a(z)|0\rangle$.

For our purpose, we need to consider the VOA with a conformal vector $\omega$, which is nothing but the chiral part $T(z)$ of the stress tensor. The modes in the expansion of $T(z)=$ $\sum L_{n} z^{-n-2}$ satisfy the Virasoro algebra (using the standard contour integral and OPE of $T(z))$

$$
\left[L_{n}, L_{m}\right]=(n-m) L_{n+m}+\frac{c\left(n^{3}-n\right)}{12} \delta_{n+m, 0} .
$$

The normal order product of two fields $a(z)$ and $b(z)$ is denoted as $: a b:(z)$, and its modes are

$$
(: a b:(z))_{n}=\sum_{n \leq-h_{a}} a_{n} b_{m-n}+\sum_{n>-h_{a}} b_{m-n} a_{n}
$$

In current convention we have $h_{a}=1$. Other properties of VOA can be found in [69].

Now let us review the definition of some special VOAs. A VOA is called rational if

1. V has finite number of irreducible representations $M_{j}$.

2. The normalized character $\operatorname{ch}_{j}=\operatorname{tr}_{M_{j}}\left(e^{2 \pi i \tau\left(L_{0}-\frac{c}{24}\right)}\right)$ converges to a holomorphic function on upper half plane $\mathbb{C}^{+6}$.

3. The function $\operatorname{ch}_{j}$ span a $S L_{2}(Z)$ invariant space.

A VOA is called finitely strongly generated if there is finite number of elements $a_{i} \in$ $V, i=1, \ldots, s$ such that the whole VOA is spanned by following normal order products

$$
: \partial^{k_{1}} a_{1} \ldots \partial^{k_{s}} a_{s}: \text {. }
$$

Notice that the choice of generators may not be unique and in general there are relations between the above basis. It is interesting to find a minimal generating set of a finitely strongly generated VOA.

For a VOA $V$, there exists a Li's filtration [70] which is a decreasing filtration

$$
F^{0} \supset F^{1} \supset F^{2} \supset \ldots,
$$

in which each $F^{p}$ is spanned by following states

$$
F^{p}(V)=\left\{a_{-n_{1}-1}^{i_{1}} a_{-n_{2}-1}^{i_{2}} \ldots|0\rangle, \quad \sum n_{i} \geq p\right\}
$$

\footnotetext{
${ }^{5}$ In physics literature, the mode expansion of a field takes the form $\sum a_{n} z^{-n-h}$ with $h$ the scaling dimension. In VOA literature, however, they use above convention of mode expansion so that they can consider VOA without the definition of scaling dimension.

${ }^{6}$ We use ch to denote the character of a VOA and $\chi$ to denote the character of a finite Lie algebra.
} 
then there is a graded sum of VOA

$$
G r(V)=\oplus_{p} \frac{F^{p}}{F^{p+1}}
$$

It is obvious that $F^{0}=V$, and $F^{1}$ is generated by $\left\{a_{-2} b \mid a \in V, b \in V\right\}$. Zhu's $C_{2}$ algebra is defined as

$$
R_{V}=\frac{F^{0}(V)}{F^{1}(V)}
$$

$R_{V}$ is a Poisson algebra and is finitely generated if and only if $V$ is strongly finitely generated. Moreover the image of generators of $V$ in $R_{V}$ generates $R_{V}$ as well. Notice that $R_{V}$ is in general not reduced, namely the ideal defining it would contain a nilpotent element ${ }^{7}$. The product and Poisson structure on $R_{V}$ are defined as

$$
\bar{a} \cdot \bar{b}=\overline{a_{-1} b}, \quad\{\bar{a}, \bar{b}\}=\overline{a_{0} b} .
$$

We have now an associated scheme and an associated variety defined from Zhu's $C_{2}$ algebra

$$
\tilde{X}_{V}=\operatorname{spec}\left(R_{V}\right), \quad X_{V}=\operatorname{spec}\left(\left(R_{V}\right)_{r e d}\right) .
$$

Recall that $X_{V}$ is a Poisson variety. If $X_{V}$ is smooth, one may view $X_{V}$ as a complexanalytic manifold equipped with a holomorphic Poisson structure, and for each point $x \in$ $X_{V}$, there is a well-defined symplectic $S_{x}$ leaf through $x$, which is the set of points that can be reached from $x$ by going along Hamiltonian flows. If $X_{V}$ is not necessarily smooth, let $\operatorname{Sing}\left(X_{V}\right)$ be the singular locus of $X$, and for any $k \geq 1$ define inductively $\operatorname{Sing}^{k}\left(X_{V}\right):=$ $\operatorname{Sing}\left(\operatorname{Sing}^{k-1}\left(X_{V}\right)\right)$. We get a finite partition

$$
X_{V}=\cup_{k} X_{V}^{k},
$$

where the strata $X_{V}^{k}:=\operatorname{Sing}^{k-1}\left(X_{V}\right) \operatorname{Sing}^{k}\left(X_{V}\right)$ are smooth analytic varieties. It is known that each $X_{V}^{k}$ inherits a Poisson structure. So for any point of $x \in X_{V}^{k}$ there is a welldefined symplectic leaf $S_{x} \subset X_{V}^{k}$. In this way one defines symplectic leaves on an arbitrary Poisson variety.

A lisse VOA is defined as the VOA such that $\operatorname{dim}\left(X_{V}\right)=0$. A rational VOA has to be lisse, but it is an open problem to prove that lisse VOA has to be rational. A quasi-lisse VOA is defined as the VOA whose associated variety $X_{V}$ has finite number of symplectic leaves. Quasi-lisse VOA has many interesting properties [31, 71]:

- The VOA is strongly finitely generated.

- The Virasoro vector $\omega_{V}$ is nilpotent in $R_{V}$.

- There are finite number of ordinary modules, and they transform nicely under modular transformations. A weak $V$-module $\left(M, Y_{M}\right)$ is called ordinary if $L_{0}$ acts semisimply on $M$, any $L_{0}$-eigenspace $M_{\Delta}$ of $M$ of eigenvalue $\Delta \in \mathbb{C}$ is finite-dimensional, and for any $\Delta \in \mathbb{C}, M_{\Delta-n}=0$ for all sufficiently large $n \in \mathbb{Z}$.

- The character satisfies a modular differential equation.

\footnotetext{
${ }^{7}$ A nilpotent element $x$ of an ideal is an element not in $I$ but $x^{n} \in I$ for some $n$.
} 


\section{$2.24 \mathrm{~d} / 2 \mathrm{~d}$ correspondence}

It was proposed in [5] that one can get a $2 \mathrm{~d}$ VOA from the Schur sector of a $4 \mathrm{~d} \mathcal{N}=2$ $\mathrm{SCFT}$, and the basic $4 \mathrm{~d} / 2 \mathrm{~d}$ dictionary used in current paper is [5]:

- There is an AKM subalgebra $\left(V^{k_{2 d}}(\mathfrak{g})\right)$ in $2 \mathrm{~d}$ VOA, where $\mathfrak{g}$ is the Lie algebra of four dimensional flavor symmetry $G_{F}$.

- The $2 \mathrm{~d}$ central charge $c_{2 d}$ and the level of AKM algebra $k_{2 d}$ are related to the $4 \mathrm{~d}$ central charge $c_{4 d}$ and the flavor central charge $k_{F}$ as

$$
c_{2 d}=-12 c_{4 d}, \quad k_{2 d}=-k_{F}^{8} .
$$

- The (normalized) vacuum character of $2 \mathrm{~d}$ VOA is the $4 \mathrm{~d}$ Schur index $\mathcal{I}(q)$.

- The associated variety is the Higgs branch of the $4 \mathrm{~d} \mathcal{N}=2 \operatorname{SCFT}[29,31,66]$.

\subsection{Comments on constraints of $2 \mathrm{~d}$ VOAs corresponding to 4d SCFTs}

It is conjectured that the VOA corresponding to a $4 \mathrm{~d} \mathcal{N}=2$ SCFT is always a lisse VOA [31]. However, not all lisse VOA has a $4 \mathrm{~d} \mathcal{N}=2$ SCFT counterpart. We do have some constraints based on $4 \mathrm{~d}$ unitarity:

- The $2 \mathrm{~d}$ central charge is negative and has to satisfy the constraint $c_{2 d} \leq-\frac{11}{30}$ for interacting $4 \mathrm{~d} \mathcal{N}=2 \operatorname{SCFTs}[72]$.

- If $4 \mathrm{~d}$ theory has a flavor group $G$, its level is bounded from below $k_{G} \geq k_{\text {critical }}[5]$, so the corresponding $2 \mathrm{~d}$ AKM level is also constrained.

- The minimal conformal weight of primary fields of VOA is constrained to be $\frac{c_{2 d}}{8} \leq$ $h_{\min } \leq 0[31]$.

These constraints come from considerations purely on the Schur sector. On the other hand, there are some very mysterious relations between the Schur sector and the Coulomb branch data:

1. First, one can compute the central charge $a_{4 d}$ and $c_{4 d}$ purely from Coulomb branch data. $c_{4 d}$ is obviously related to the $2 \mathrm{~d} \mathrm{VOA}$, and $a_{4 d}-c_{4 d}$ is also related to the asymptotic limit of the Schur index, see [31] and further discussions in section 4.5.

2. One can compute the Schur index from the Coulomb branch massive BPS spectrum $[9,13]$.

3. If we know the common denominator $r$ of Coulomb branch operators, the flavor central charge seem to be bounded by a number which depends on the denominator $r$ [73]. This bound is different from the minimal bound found from Higgs branch data only.

\footnotetext{
${ }^{8}$ Our normalization of $k_{F}$ is half of that of $[5,6]$.
} 
So from this perspective, the bound from purely Higgs branch data seems to be not enough on constraining the set of quasi-lisse VOA which can be VOA of $4 \mathrm{~d}$ theory. With input from Coulomb branch data, one can get much stronger constraint on VOA, and we plan to study this further in the near future.

\section{$3 \quad$ Argyres-Douglas theories and $W$-algebras}

In this section we review known results on the classification of $\mathrm{AD}$ theories from $M 5$ branes and their corresponding VOAs. We focus on $\mathrm{AD}$ theories whose VOAs are $W$-algebras at boundary admissible levels.

\subsection{AD theories correspond to $W^{k^{\prime}}(\mathfrak{g}, f)$ algebras}

One can engineer a large class of four dimensional $\mathcal{N}=2$ SCFTs by starting from a $6 \mathrm{~d}$ $(2,0)$ theory of type $\mathfrak{j}=A D E$ on a sphere with an irregular singularity and a regular singularity[39, 57, 58, 74, 75]. The Coulomb branch is captured by a Hitchin system with singular boundary conditions near the singularity. The Higgs field of the Hitchin system near the irregular singularity takes the following form

$$
\Phi=\frac{T}{z^{2+\frac{k}{b}}}+\ldots,
$$

where $T$ is determined by a positive principle grading of Lie algebra $\mathfrak{j}$ [76], and is a regular semi-simple element of $\mathbf{j} . k$ is an integer greater than $b$. Subsequent terms are chosen such that they are compatible with the leading order term (essentially the grading determines the choice of these terms). We call them $J^{(b)}[k]$ type irregular puncture. Theories constructed using only above irregular singularities can also be engineered using a three dimensional singularity in type IIB string theory as summarized in table 1[77].

One can add another regular singularity which is labeled by a nilpotent orbit $f$ of $\mathfrak{j}$ (We use Nahm labels such that the trivial orbit corresponding to a regular puncture with maximal flavor symmetry). A detailed discussion on these defects can be found in [78].

To get non-simply laced flavor groups, we need to consider the outer-automorphism twist of ADE Lie algebra and its Langlands dual. A systematic study of these AD theories was performed in [39]. Denoting the twisted Lie algebra of $\mathfrak{j}$ as $\mathfrak{g}^{\vee}$ and its Langlands dual as $\mathfrak{g}$, outer-automorphisms and twisted algebras of $\mathfrak{j}$ are summarized in table 2 . The irregular singularity of regular semi-simple type is also classified in table 3 with the following form

$$
\Phi=\frac{T^{t}}{z^{2+\frac{k}{b}}}+\ldots
$$

Here $T^{t}$ is an element of Lie algebra $\mathfrak{g}^{\vee}$ or other parts of the decomposition of $\mathfrak{j}$ under outer automorphism. $k>-b$, and the novel thing is that $k$ could take half-integer value or in thirds $\left(\mathfrak{g}=G_{2}\right)$. One can also represent those irregular singularities by 3 -fold singularities as in table 3 .

We could again add a twisted regular puncture labeled also by a nilpotent orbit $f$ of $\mathfrak{g}$. If there is no mass parameter in the irregular singularity, the corresponding VOA is given 


\begin{tabular}{|c|c|c|}
\hline $\mathfrak{j}$ & $b$ & Singularity \\
\hline$A_{N-1}$ & $N$ & $x_{1}^{2}+x_{2}^{2}+x_{3}^{N}+z^{k}=0$ \\
\hline & $N-1$ & $x_{1}^{2}+x_{2}^{2}+x_{3}^{N}+x_{3} z^{k}=0$ \\
\hline$D_{N}$ & $2 N-2$ & $x_{1}^{2}+x_{2}^{N-1}+x_{2} x_{3}^{2}+z^{k}=0$ \\
\hline & $N$ & $x_{1}^{2}+x_{2}^{N-1}+x_{2} x_{3}^{2}+z^{k} x_{3}=0$ \\
\hline$E_{6}$ & 12 & $x_{1}^{2}+x_{2}^{3}+x_{3}^{4}+z^{k}=0$ \\
\hline & 9 & $x_{1}^{2}+x_{2}^{3}+x_{3}^{4}+z^{k} x_{3}=0$ \\
\hline & 8 & $x_{1}^{2}+x_{2}^{3}+x_{3}^{4}+z^{k} x_{2}=0$ \\
\hline$E_{7}$ & 18 & $x_{1}^{2}+x_{2}^{3}+x_{2} x_{3}^{3}+z^{k}=0$ \\
\hline & 14 & $x_{1}^{2}+x_{2}^{3}+x_{2} x_{3}^{3}+z^{k} x_{3}=0$ \\
\hline$E_{8}$ & 30 & $x_{1}^{2}+x_{2}^{3}+x_{3}^{5}+z^{k}=0$ \\
\hline & 24 & $x_{1}^{2}+x_{2}^{3}+x_{3}^{5}+z^{k} x_{3}=0$ \\
\hline & 20 & $x_{1}^{2}+x_{2}^{3}+x_{3}^{5}+z^{k} x_{2}=0$ \\
\hline
\end{tabular}

Table 1: Three-fold isolated quasi-homogenous singularities of cDV type corresponding to the $J^{(b)}[k]$ irregular punctures of the regular-semisimple type in [58]. These 3d singularity is very useful in extracting the Coulomb branch spectrum[77].

\begin{tabular}{|c|c|c|c|c|c|}
\hline$j$ & $A_{2 N}$ & $A_{2 N-1}$ & $D_{N+1}$ & $E_{6}$ & $D_{4}$ \\
\hline Outer-automorphism $o$ & $Z_{2}$ & $Z_{2}$ & $Z_{2}$ & $Z_{2}$ & $Z_{3}$ \\
\hline Invariant subalgebra $\mathfrak{g}^{\vee}$ & $B_{N}$ & $C_{N}$ & $B_{N}$ & $F_{4}$ & $G_{2}$ \\
\hline Flavor symmetry $\mathfrak{g}$ & $C_{N}^{(1)}$ & $B_{N}$ & $C_{N}^{(2)}$ & $F_{4}$ & $G_{2}$ \\
\hline
\end{tabular}

Table 2: Outer-automorphisms of simple Lie algebras $j$, its invariant subalgebra $g^{\vee}$ and flavor symmetry $g$ from the Langlands dual of $g^{\vee}$.

by the following $W$ algebra[39]

$$
W^{k^{\prime}}(\mathfrak{g}, f), \quad k^{\prime}=-h^{\vee}+\frac{1}{n} \frac{b}{k+b}
$$

where $h^{\vee}$ is the dual Coxeter number of $\mathfrak{g}, n$ is the number listed in table 4 , and $k$ is restricted to the value such that no mass parameter is in the irregular singularity.

In this paper, we are going to focus on the choice of $b$ and $n$ such that the corresponding $W$ algebra takes the following form

$$
W^{k^{\prime}}(\mathfrak{g}, f), \quad k^{\prime}=-h^{\vee}+\frac{h^{\vee}}{k+h^{\vee}}, \quad\left(k, h^{\vee}\right)=1 .
$$

There are some further constraints on value $k$ : a) $h^{\vee}+k \geq 2$; b) $k$ and $h^{\vee}$ coprime; and c) $k \neq 2 n$ for $\mathfrak{g}=B_{N}, C_{N}, F_{4}$, and $k \neq 3 n$ for $\mathfrak{g}=G_{2}$. 


\begin{tabular}{|c|c|c|c|}
\hline$j$ with twist & $b_{t}$ & SW geometry at SCFT point & $\Delta[z]$ \\
\hline$A_{2 N} / Z_{2}$ & $4 N+2$ & $x_{1}^{2}+x_{2}^{2}+x^{2 N+1}+z^{k+\frac{1}{2}}=0$ & $\frac{4 N+2}{4 N+2 k+3}$ \\
\hline & $2 N$ & $x_{1}^{2}+x_{2}^{2}+x^{2 N+1}+x z^{k}=0$ & $\frac{2 N}{k+2 N}$ \\
\hline$A_{2 N-1} / Z_{2}$ & $4 N-2$ & $x_{1}^{2}+x_{2}^{2}+x^{2 N}+x z^{k+\frac{1}{2}}=0$ & $\frac{4 N-2}{4 N+2 k-1}$ \\
\hline & $2 N$ & $x_{1}^{2}+x_{2}^{2}+x^{2 N}+z^{k}=0$ & $\frac{2 N}{2 N+k}$ \\
\hline$D_{N+1} / Z_{2}$ & $2 N+2$ & $x_{1}^{2}+x_{2}^{N}+x_{2} x_{3}^{2}+x_{3} z^{k+\frac{1}{2}}=0$ & $\frac{2 N+2}{2 k+2 N+3}$ \\
\hline & $2 N$ & $x_{1}^{2}+x_{2}^{N}+x_{2} x_{3}^{2}+z^{k}=0$ & $\frac{2 N}{k+2 N}$ \\
\hline$D_{4} / Z_{3}$ & 12 & $x_{1}^{2}+x_{2}^{3}+x_{2} x_{3}^{2}+x_{3} z^{k \pm \frac{1}{3}}=0$ & $\frac{12}{12+3 k \pm 1}$ \\
\hline & 6 & $x_{1}^{2}+x_{2}^{3}+x_{2} x_{3}^{2}+z^{k}=0$ & $\frac{6}{6+k}$ \\
\hline$E_{6} / Z_{2}$ & 18 & $x_{1}^{2}+x_{2}^{3}+x_{3}^{4}+x_{3} z^{k+\frac{1}{2}}=0$ & $\frac{18}{18+2 k+1}$ \\
\hline & 12 & $x_{1}^{2}+x_{2}^{3}+x_{3}^{4}+z^{k}=0$ & $\frac{12}{12+k}$ \\
\hline & 8 & $x_{1}^{2}+x_{2}^{3}+x_{3}^{4}+x_{2} z^{k}=0$ & $\frac{8}{12+k}$ \\
\hline
\end{tabular}

Table 3: Seiberg-Witten geometry of twisted theories at the SCFT point.

\begin{tabular}{|c|c|c|c|c|}
\hline & dimension & $h$ & $h^{\vee}$ & $n$ \\
\hline$A_{N-1}$ & $N^{2}-1$ & $N$ & $N$ & 1 \\
\hline$B_{N}$ & $(2 N+1) N$ & $2 N$ & $2 N-1$ & 2 \\
\hline$C_{N}^{(1)}$ & $(2 N+1) N$ & $2 N$ & $N+1$ & 4 \\
\hline$C_{N}^{(2)}$ & $(2 N+1) N$ & $2 N$ & $N+1$ & 2 \\
\hline$D_{N}$ & $N(2 N-1)$ & $2 N-2$ & $2 N-2$ & 1 \\
\hline$E_{6}$ & 78 & 12 & 12 & 1 \\
\hline$E_{7}$ & 133 & 18 & 18 & 1 \\
\hline$E_{8}$ & 248 & 30 & 30 & 1 \\
\hline$F_{4}$ & 52 & 12 & 9 & 2 \\
\hline$G_{2}$ & 14 & 6 & 4 & 3 \\
\hline
\end{tabular}

Table 4: Lie algebra data. $h$ is the Coxeter number and $h^{\vee}$ is the dual Coxeter number.

\section{The character of $W$-algebra and the Schur index}

Now we discuss Schur indices of AD theories from their corresponding $W$-algebras. The index can be written in a simplified form which implies many interesting properties of the SCFT and the VOA.

\subsection{The $W$-algebra from the qDS reduction}

We first set up the notation for Lie algebra datas. Let $\mathfrak{g}$ be a simple finite dimensional Lie algebra, and let $\mathfrak{h}$ be the Cartan subalgebra of $\mathfrak{g}$, and let $\Delta \subset \mathfrak{h}^{*}$ be the set of roots, where $\mathfrak{h}^{*}$ is the dual space of $\mathfrak{h}$. Let $Q=\mathbb{Z} \Delta$ be the root lattice and let $Q^{*}=\{h \in \mathfrak{h} \mid \alpha(h) \in$ $\mathbb{Z}$ for all $\alpha \in \Delta\}$ be its dual lattice. We also use $\Delta_{+}$to denote the set of positive roots, and $\left\{\alpha_{1}, \ldots, \alpha_{l}\right\}$ be the set of simple roots with $l$ be the rank of $\mathfrak{g}$. We denote $\rho$ as the half 
of the sum of all positive roots. The bracket $(\cdot \mid \cdot)$ is the invariant bilinear form on $\mathfrak{g}$ with the normalization $(\alpha \mid \alpha)=2$ for the long roots. $h^{\vee}$ is the dual Coxeter number. We use $\omega_{i}$ to denote the fundamental weights of Lie algebra $\mathfrak{g}$.

Now for AKM algebra $\hat{\mathfrak{g}}=\mathfrak{g}\left[t, t^{-1}\right]+C K+C d$, its Cartan subalgebra is $\hat{\mathfrak{h}}=\mathfrak{h}+C K+C d$. The bilinear form on AKM algebra is extended from the bilinear form of $\mathfrak{g}$ as follows

$$
(\mathfrak{h} \mid C K+C d)=0,(K \mid K)=0,(d \mid d)=0,(d \mid K)=1 .
$$

We can use this bilinear form to identify the dual space $\hat{h}^{*}$ with $\hat{h}$. Roots of AKM are denoted by three sets of eigenvalues. The imaginary root has the label $\delta=(0,0,1)$ and simple roots are $\hat{\alpha}_{i}=\left(\alpha_{i}, 0,0\right)$ with $\alpha_{i}$ being simple roots of $\mathfrak{g}$. Furthermore we have the zeroth simple root $\hat{\alpha}_{0}=(-\theta, 0,1)$ with $\theta$ being the highest root of $\mathfrak{g}$. The set of real roots are $\hat{\Delta}^{r e}=\{\alpha+n \delta \mid \alpha \in \Delta, n \in \mathbb{Z}\}$, and the set of positive real roots is denoted as $\hat{\Delta}_{+}^{r e}=\Delta_{+} \cup\left\{\alpha+n \delta \mid \alpha \in \Delta, n \in \mathbb{Z}_{\geq 1}\right\}$. Affine fundamental weights are $\Lambda_{0}=(0,1,0)$ and $\Lambda_{i}=\left(\omega_{i}, a_{i}^{\vee}, 0\right)$ with $a_{i}^{\vee}$ being the comark which is 1 for simply laced Lie algebra. We also define $\hat{\rho}=\sum_{i=0}^{l} \hat{\omega}_{i}$. One has following important set of roots

$$
\hat{\Pi}_{u}=\left\{u \delta-\theta, \hat{\alpha}_{1}, \ldots, \hat{\alpha}_{l}\right\}
$$

which is used in defining principle admissible weights.

For a $\beta \in Q^{*}$, one define a translation $t_{\beta} \in \operatorname{End}\left(\hat{h}^{*}\right)$ with the following formula

$$
t_{\beta}(\lambda)=\lambda+\lambda(K) \beta-\left((\lambda, \beta)+\frac{1}{2} \lambda(K)|\beta|^{2}\right) \delta .
$$

An element in the extended affine Weyl group $\tilde{W}$ can be written in the form $t_{\beta} y$ with $y \in W$ an element in Weyl group of lie algebra $\mathfrak{g}$.

Now $\Lambda$ is called a principle admissible weight if the following two properties hold

1. The level $k=\Lambda(K)$ is a rational number with denominator $u \in \mathbb{Z}_{\geq 1}$, such that

$$
k+h^{\vee} \geq \frac{h^{\vee}}{u} \text { and } \operatorname{gcd}\left(u, h^{\vee}\right)=\operatorname{gcd}\left(u, r^{\vee}\right)=1,
$$

where $r^{\vee}$ takes 1 for $\mathfrak{g}$ of type ADE, and 2 for $\mathfrak{g}$ of type B, C, F, and 3 for $\mathfrak{g}=G_{2}$.

2. All principal admissible weights $\Lambda$ are of the form

$$
\Lambda=\left(t_{\beta} y\right) \cdot\left(\Lambda^{0}-(u-1)\left(k+h^{\vee}\right) \Lambda_{0}\right)
$$

where $\beta \in Q^{*}, y \in W$ are such that $\left(t_{\beta} y\right) \hat{\Pi}_{u} \subset \hat{\Delta}_{+}, \Lambda^{0}$ is an integrable weight of level $u\left(k+h^{\vee}\right)-h^{\vee}$, and dot denotes the shifted action $w \cdot \Lambda=w(\Lambda+\hat{\rho})-\hat{\rho}$.

Starting with an AKM algebra $V^{k}(\mathfrak{g})$, one can get a large class of $W$ algebras by using the quantum Drinfeld-Soklov reduction [60]. Given a $\mathfrak{s l}_{2}$ triple $(x, e, f)$ with the nilpotent element $f$, and the commutation relation is defined as

$$
[x, e]=e, \quad[x, f]=-f, \quad[e, f]=2 x .
$$


The corresponding $W$ algebra is denoted as $W^{k}(\mathfrak{g}, f)$. The universal $W$ algebra has following properties: it is finitely strongly generated by the following fields $J_{v_{j}}$ with scaling dimension $1-j$. Here $v_{j} \in \mathfrak{g}_{j}^{f}$ with $j \leq 0$. Let's explain the notation now: Given a $\mathfrak{s l}_{2}$ triple $(x, e, f)$ with $x$ a semi-simple element, we can decompose $\mathfrak{g}$ as: $\mathfrak{g}=\oplus \mathfrak{g}_{j}$ with $\mathfrak{g}_{j}=\left\{\left[x, g_{j}\right]=j g_{j}\right\} \cdot \mathfrak{g}_{j}^{f}$ is defined as the elements in $\mathfrak{g}_{j}$ which also commutes with nilpotent element $f$. There is a symmetry between $\pm j \operatorname{such}$ that $\operatorname{dim} \mathfrak{g}_{j}^{f}=\operatorname{dim} \mathfrak{g}_{-j}^{f}$.

\subsection{Character of $W$-algebra modules}

For admissible modules of AKM and corresponding W-algebras at boundary level, their characters decompose in products in terms of the Jacobi form $\theta_{11}(\tau, z)[62]$. This result provides an elegant closed form formula for Schur indices of the AD theory discussed in this paper.

Starting with AKM at boundary level $k=-h^{\vee}+\frac{h^{\vee}}{u}$, all boundary principal admissible weights are of the form

$$
\Lambda=\left(t_{\beta} y\right) \cdot\left(k \Lambda_{0}\right),
$$

where $\beta \in Q^{*}, y \in W$ are such that $\left(t_{\beta} y\right) \hat{\Pi}_{u} \subset \hat{\Delta}_{+}$. The character of the module corresponding to admissible weight $\Lambda$ can be expressed in products of theta functions [62]

$$
\operatorname{ch}_{\Lambda}(\tau, z, t)=e^{2 \pi i\left(k t+\frac{h^{\vee}}{u}(z \mid \beta)\right)} q^{\frac{h^{\vee}}{2 u}|\beta|^{2}}\left(\frac{\eta(u \tau)}{\eta(\tau)}\right)^{\frac{1}{2}(3 l-\operatorname{dim} \mathfrak{g})} \prod_{\alpha \in \Delta_{+}} \frac{\theta_{11}(y(\alpha)(z+\tau \beta), u \tau)}{\theta_{11}(\alpha(z), \tau)} .
$$

Convention of $\eta(\tau)$ and $\theta_{11}$ are summarized in appendix A. In particular the vacuum module has the weight $k \Lambda_{0}$, and its character is

$$
\operatorname{ch}_{k \Lambda_{0}}(\tau, z, t)=e^{2 \pi i k t}\left(\frac{\eta(u \tau)}{\eta(\tau)}\right)^{\frac{1}{2}(3 l-\operatorname{dim} \mathfrak{g})} \prod_{\alpha \in \Delta_{+}} \frac{\theta_{11}(\alpha(z), u \tau)}{\theta_{11}(\alpha(z), \tau)} .
$$

The Schur index of the corresponding AD theory is obtained simply by setting $t=0$ and normalizing the character such that the Schur index goes to one when $q=e^{2 \pi i \tau}$ goes to zero.

For W-algebra $W^{k}(\mathfrak{g}, f)$ from the vacuum $\hat{\mathfrak{g}}$-module of level $k$ by the qDS reduction, there is a reductive functor $H$ which maps principle admissible modules of AKM to either zero or an irreducible module of $W^{k}(\mathfrak{g}, f)$. The character of the irreducible $W^{k}(\mathfrak{g}, f)$ module $H(\Lambda)$ is

$$
\begin{aligned}
\operatorname{ch}_{H(\Lambda)}(\tau, z)= & (-i)^{\left|\Delta_{+}\right|} q^{\frac{h^{\vee}}{2 u}|\beta-x|^{2}} e^{\frac{2 \pi i h^{\vee}}{u}(\beta \mid z)} \\
& \times \frac{\eta(u \tau)^{\frac{3}{2} l-\frac{1}{2} \operatorname{dim} \mathfrak{g}}}{\eta(\tau)^{\frac{3}{2} l-\frac{1}{2} \operatorname{dim}\left(\mathfrak{g}_{0}+\mathfrak{g}_{\frac{1}{2}}\right)}} \frac{\prod_{\alpha \in \Delta_{+}} \theta_{11}(y(\alpha)(z+\tau \beta-\tau x), u \tau)}{\prod_{\alpha \in \Delta_{+}^{0}} \theta_{11}(\alpha(z), \tau)\left(\prod_{\alpha \in \Delta_{\frac{1}{2}}} \theta_{01}(\alpha(z), \tau)\right)^{\frac{1}{2}}},
\end{aligned}
$$

where $\{f, x, e\}$ forms the $\mathfrak{s l}_{2}$-triple in $\mathfrak{g}, \mathfrak{g}=\oplus_{j} \mathfrak{g}_{j}$ is the eigenspace decomposition for ad $x$, $\Delta_{j} \subset \Delta$ is the set of roots of the root spaces in $\mathfrak{g}_{j}$ and $\Delta_{+}^{0}=\Delta_{+} \cap \Delta_{0}$. If the reduction of 
$\Lambda$ gives zero, $\operatorname{ch}_{H(\Lambda)}=0$ automatically. If $\Lambda_{1}$ and $\Lambda_{2}$ lead to the same module in the $\mathrm{W}$ algebra, $\operatorname{ch}_{H\left(\Lambda_{1}\right)}=\operatorname{ch}_{H\left(\Lambda_{2}\right)}$. In particular the vacuum module of the $\mathrm{W}$-algebra is $H\left(k \Lambda_{0}\right)$ with the character

$$
\operatorname{ch}_{H\left(k \Lambda_{0}\right)}(\tau, z)=(-i)^{\left|\Delta_{+}\right|} q^{\frac{h^{\vee}}{2 u}|x|^{2}} \frac{\eta(u \tau)^{\frac{3}{2} l-\frac{1}{2} \operatorname{dim} \mathfrak{g}}}{\eta(\tau)^{\frac{3}{2} l-\frac{1}{2} \operatorname{dim}\left(\mathfrak{g}_{0}+\mathfrak{g}_{\frac{1}{2}}\right)}} \frac{\prod_{\alpha \in \Delta_{+}} \theta_{11}(\alpha(z-\tau x), u \tau)}{\prod_{\alpha \in \Delta_{+}^{0}} \theta_{11}(\alpha(z), \tau)\left(\prod_{\alpha \in \Delta_{\frac{1}{2}}} \theta_{01}(\alpha(z), \tau)\right)^{\frac{1}{2}}} .
$$

It also gives the Schur index of the corresponding AD theory after normalization.

\subsection{The simplified form}

Using the product formula in the previous section, we can put the index of $W^{k}(\mathfrak{g}, f)$ in a even simpler form. If $f$ is regular principle, the index is thus

$$
\mathcal{I}_{W^{k^{\prime}}\left(\mathfrak{g}, f_{\text {prin }}\right)}=\operatorname{PE}\left[\frac{\sum_{i} q^{d_{i}}-q^{k-1}\left(\sum q^{d_{i}}\right)}{(1-q)\left(1-q^{h^{\vee}+k}\right)}\right], \quad k^{\prime}=-h^{\vee}+\frac{h^{\vee}}{h^{\vee}+k},
$$

where $h^{\vee}$ is the dual Coxeter number, the plethystic exponential $P E$ is defined as

$$
P E[f(a, \cdots)]=\exp \left[\sum_{n=1}^{\infty} \frac{1}{n} f\left(a^{n}, \cdots\right)\right],
$$

and $\left\{d_{i}\right\}$ is the set of exponents of Lie algebra $\mathfrak{g}$. On the other hand, if $f$ is trivial, the index becomes

$$
\mathcal{I}_{W^{k^{\prime}}\left(\mathfrak{g}, f_{t r i}\right)}=P E\left[\frac{q-q^{h^{\vee}+k}}{(1-q)\left(1-q^{h^{\vee}+k}\right)} \chi_{a d j}(z)\right], \quad k^{\prime}=-h^{\vee}+\frac{h^{\vee}}{h^{\vee}+k},
$$

where $\chi_{\text {adj }}(z)$ is the character of the adjoint representation of $\mathfrak{g}$. For generic $f$, the Lie group $G$ of $\mathfrak{g}$ has a subgroup $S U(2) \times G_{F}$ with $G_{F}$ being the flavor group determined by $f$. Under this subgroup the adjoint representation of $G$ decomposes as

$$
\operatorname{adj}_{G}=\sum_{j} V_{j} \otimes R_{j}
$$

where $V_{j}$ is the spin $j$ representation of $S U(2)$ and $R_{j}$ is the corresponding representation of $G_{F}$. The Schur index takes the following form $\left(k^{\prime}=-h^{\vee}+\frac{h^{\vee}}{h^{\vee}+k}\right)$

$$
\mathcal{I}_{W^{k^{\prime}(\mathfrak{g}, f)}}=P E\left[\sum \frac{q^{1+j}}{1-q} \chi_{R_{j}}(z)-\frac{q^{h^{\vee}+k}}{1-q^{h^{\vee}+k}} \sum_{j} \chi_{V_{j}}(q) \chi_{R_{j}}(z)\right]
$$

with $\chi_{V_{j}}$ being the character of spin $j$ representation of $S U(2)$ and $\chi_{R_{j}}(z)$ being the character of $R_{j}$ defined as $\operatorname{tr}_{R_{j}} e^{2 \pi i z}$ with $z \in \mathfrak{h}^{f}$ and $\mathfrak{h}^{f}$ are the Cartan of $\mathfrak{g}_{0}^{f}$ (centralizer of the $\mathfrak{s l}_{2}$ triple $(x, e, f)$ ). The dimension of $R_{j}$ is the same as the dimension of $\mathfrak{g}_{ \pm j}^{f}$. We rewrite the character as follows

$\mathcal{I}_{W^{k^{\prime}}(\mathfrak{g}, f)}=P E\left[\frac{\sum_{j} q^{1+j} \chi_{R_{j}}(z)}{(1-q)\left(1-q^{h^{\vee}+k}\right)}-\frac{q^{h^{\vee}+k}}{(1-q)\left(1-q^{h^{\vee}+k}\right)}\left[\sum q^{1+j} \chi_{R_{j}}(z)+(1-q) \sum_{j} \chi_{V_{j}}(q) \chi_{R_{j}}(z)\right]\right]$. 
To further simplify this expression, we use the following identity

$$
\chi_{V_{j}}(q)=\frac{q^{-j-1 / 2}-q^{j+1 / 2}}{q^{-1 / 2}-q^{1 / 2}}=\frac{q^{-j}-q^{j+1}}{1-q},
$$

and finally our index for generic $f$ takes the following form

$$
\mathcal{I}_{W^{k^{\prime}}(g, f)}=P E\left[\frac{\sum_{j} q^{1+j} \chi_{R_{j}}(z)-q^{h^{\vee}+k} \sum_{j} q^{-j} \chi_{R_{j}}(z)}{(1-q)\left(1-q^{h^{\vee}+k}\right)}\right] .
$$

\subsubsection{Applications}

Level-Rank duality: One can check the level-rank duality using the index formula 4.12. For example, the Schur index of $\left(A_{N-1}, A_{k-1}\right)$ AD theory is

$$
\mathcal{I}_{\left(A_{N-1}, A_{k-1}\right)}=P E\left[\frac{\left(1-q^{k-1}\right) \sum_{j=2}^{N} q^{j}}{(1-q)\left(1-q^{N+k}\right)}\right]=P E\left[\frac{q^{2}\left(1-q^{k-1}\right)\left(1-q^{N-1}\right)}{(1-q)^{2}\left(1-q^{N+k}\right)}\right],
$$

which is symmetric under the exchange of $k$ and $N$, reproducing the result in [29]. For $\left(G_{1}, G_{2}\right)$ theories with $\operatorname{gcd}\left(h_{1}^{\vee}, h_{2}^{\vee}\right)=1$, the Schur index can also be written as

$$
\mathcal{I}_{\left(G_{1}, G_{2}\right)}=P E\left[\frac{\left(\sum_{i} q^{d_{i}^{(1)}}\right)\left(\sum_{j} q^{d_{j}^{(2)}}\right)}{q^{2}\left(1-q^{h_{1}^{\vee}+h_{2}^{\vee}}\right)}\right],
$$

where $d_{i}^{(r)}$ 's are degrees of Casimirs of $G_{r}$.

This type of level rank duality is vastly generalized in [56]. One example is the following identification of $W$-algebras

$$
\begin{aligned}
& W^{-n 1(n+k)-n+\frac{n_{1}(n+k)+n}{n+k}}\left(\mathfrak{s l}_{n_{1}(n+k)+n},\left[(n+k-1)^{n_{1}}, n+n_{1}\right]\right) \\
& =W^{-k+\frac{k}{n+k}}\left(\mathfrak{s l}_{k},\left[k-n_{1}, 1^{n_{1}}\right]\right) .
\end{aligned}
$$

To compute the Schur index of the RHS, notice that the character of the adjoint representation of $\mathfrak{s l}_{k}$ decomposes under $\left[k-n_{1}, 1^{n_{1}}\right]$ as

$$
\chi_{a d j}^{\mathfrak{s} l_{k}}=\chi_{a d j}+\left(a^{2 n_{1}-k} \chi_{\square}+a^{-2 n_{1}+k} \chi_{\square}\right) \chi_{\frac{V_{\frac{k-n_{1}-1}{2}}}{2}}+\sum_{j=0}^{k-n_{1}-1} \chi_{V_{j}},
$$

where $\chi_{a d j}, \chi_{\square}$ and $\chi_{\bar{\square}}$ are the characters of adjoint, fundamental and anti-fundamental representations of $S U\left(n_{1}\right)$ respectively. Therefore the Schur index for the RHS follows the simplified formula 4.19

$$
\mathcal{I}_{R H S}=P E\left[\frac{f_{R H S}}{(1-q)\left(1-q^{n+k}\right)}\right]
$$

where $f_{R H S}$ is

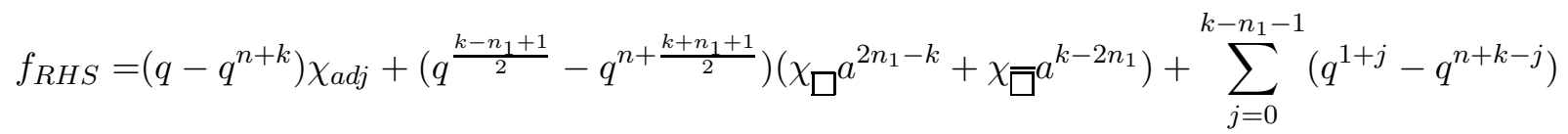

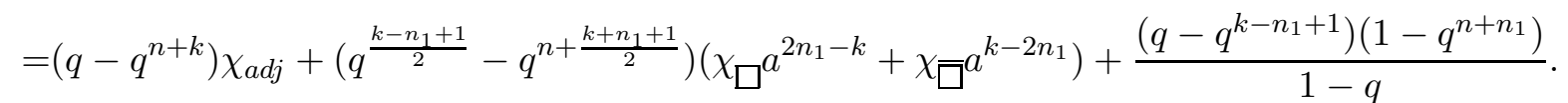


On the other hand, the character of the adjoint representations of $\mathfrak{s l}_{n_{1}(n+k)+n}$ decomposes under $\left[(n+k-1)^{n_{1}}, n+n_{1}\right]$ as

$$
\begin{aligned}
\chi^{\mathfrak{s l}_{n_{1}(n+k)+n}=} & \left(\chi_{a d j}+1\right) \sum_{j=0}^{n+k-2} \chi_{V_{j}}+\sum_{j=1}^{n+n_{1}-1} \chi_{V_{j}} \\
& +\left(b^{n-n_{1}(n+k)} \chi_{\square}+b^{n_{1}(n+k)-n} \chi_{\bar{\square}}\right) \sum_{j=\left(k-n_{1}-1\right) / 2}^{n+\left(n_{1}+k-3\right) / 2} \chi_{V_{j}},
\end{aligned}
$$

hence the Schur index for the LHS is

$$
\mathcal{I}_{L H S}=P E\left[\frac{f_{L H S}}{(1-q)\left(1-q^{n+k}\right)}\right]
$$

with

$$
\begin{aligned}
f_{L H S}= & \left(\sum_{j=0}^{n+k-2} q^{1+j}-q^{n+k-j}\right)\left(\chi_{a d j}+1\right)+\sum_{j=1}^{n+n_{1}-1}\left(q^{1+j}-q^{n+k-j}\right) \\
& +\left(b^{n-n_{1}(n+k)} \chi_{\square}+b^{n_{1}(n+k)-n} \chi_{\bar{\square}}\right) \sum_{j=\left(k-n_{1}-1\right) / 2}^{n+\left(n_{1}+k-3\right) / 2}\left(q^{1+j}-q^{n+k-j}\right) \\
= & \left(q-q^{n+k}\right) \chi_{a d j}+\frac{\left(q-q^{k-n_{1}+1}\right)\left(1-q^{n+n_{1}}\right)}{1-q} \\
& +\left(q^{\frac{k-n_{1}+1}{2}}-q^{n+\frac{k+n_{1}+1}{2}}\right)\left(b^{n-n_{1}(n+k)} \chi_{\square}+b^{n_{1}(n+k)-n} \chi_{\bar{\square}}\right) .
\end{aligned}
$$

Comparing equation 4.25 and 4.28 , we see $f_{L H S}=f_{R H S}$ with a redefinition of $b$, therefore $\mathcal{I}_{L H S}=\mathcal{I}_{R H S}$, providing another check of the generalized level-rank duality 4.22 .

Collapsing levels: One may also understand the phenomenon of collapsing levels $[56,79]$ by using the character formula 4.19. For example, consider $\mathfrak{g}=\mathfrak{s l}_{N}$ and the nilpotent orbit labelled by the Young tableaux $Y=\left[2,1^{N-2}\right]$. The flavor group is then $S U(N-2) \times U(1)$. The character of the adjoint representation of $\mathfrak{s l}_{N}$ decomposes under such nilpotent element as

$$
\chi_{a d j}^{\mathfrak{s l}_{N}}=\left(\chi_{a d j}+1\right)+\left(a \chi_{\square}+\frac{1}{a} \chi_{\square}\right) \chi_{V_{1 / 2}}+\chi_{V_{1}},
$$

where the fugacity $a$ is the exponential of the $U(1)$ Cartan and $\chi_{a d j}, \chi_{\square}$ and $\chi_{\bar{\square}}$ are the character of adjoint, fundamental and anti-fundamental representation of $S U(N-2)$ respectively. One sees immediately from the formula 4.19 that terms proportional to $\chi_{V_{1 / 2}}$ cancel with each other when $k+h^{\vee}=2$

$$
P E\left[\frac{q\left(\chi_{a d j}+1\right)+q^{2}-q^{2}\left(\chi_{a d j}+1\right)-q}{(1-q)\left(1-q^{2}\right)}\right]=P E\left[\frac{q \chi_{a d j}}{1-q^{2}}\right],
$$

hence the character reduces to the character of the affine $\mathfrak{s l}_{N-2}$ algebra with the level $\tilde{k}=-(N-2)+\frac{N-2}{2}$. 
For $\mathfrak{g}=\mathfrak{s l}_{N}$ and $Y=\left[r^{m}, 1^{N-r m}\right]$, the flavor symmetry is $G_{F}=S U(m) \times S U(N-r m) \times$ $U(1)$. The character of the adjoint representation of $\mathfrak{s l}_{N}$ decomposes under $S U(2) \times G_{F}$ as

$$
\begin{aligned}
\chi_{a d j}^{\mathfrak{s l}_{N}}= & \chi_{a d j}^{S U(N-r m)}+\left(\chi_{V_{0}}+\chi_{V_{1}}+\cdots+\chi_{V_{r-1}}\right)\left(\chi_{a d j}^{S U(m)}+1\right) \\
& +\left(a^{N-r m-m} \chi_{\square}^{S U(m)} \chi_{\bar{\square}}^{S U(N-r m)}+a^{m-N+r m} \chi_{\bar{\square}}^{S U(m)} \chi_{\square}^{S U(N-r m)}\right) \chi_{\frac{V_{\frac{r-1}{2}}}{\square U}},
\end{aligned}
$$

again the fugacity $a$ labels the $U(1)$ symmetry. At $k+h^{\vee}=r$, the generator and relation which contain both representations of $S U(m)$ and $S U(N-r m)$ cancel with each other and the character is

$$
\begin{aligned}
& P E\left[\frac{\left(q-q^{r}\right) \chi_{a d j}^{S U(N-r m)}}{(1-q)\left(1-q^{r}\right)}+\frac{\left(q-q^{r}\right)+\left(q^{2}-q^{r-1}\right)+\cdots+\left(q^{r}-q\right)}{(1-q)\left(1-q^{r}\right)}\left(\chi_{a d j}^{S U(m)}+1\right)\right] \\
& =P E\left[\frac{\left(q-q^{r}\right) \chi_{a d j}^{S U(N-r m)}}{(1-q)\left(1-q^{r}\right)}\right],
\end{aligned}
$$

which is the same as the vacuum character of the affine $\mathfrak{s l}_{N-r m}$ with level $\tilde{k}=-h^{\vee}+\frac{h^{\vee}}{r}$.

For $\mathfrak{g}=\mathfrak{s o}_{2 N}$ and $Y=\left[r^{m}, 1^{2 N-r m}\right]$ with $r$ being an odd number, the flavor symmetry is $G_{F}=S O(m) \times S O(2 N-r m)$. The character of the adjoint representation of $\mathfrak{s o}_{2 N}$ decomposes under $S U(2) \times G_{F}$ as

$$
\begin{aligned}
\chi_{a d j}^{\mathfrak{s o}_{2 N}}= & \chi_{a d j}^{S O(2 N-r m)}+\left(\chi_{V_{0}}+\chi_{V_{2}}+\cdots+\chi_{V_{r-1}}\right) \chi_{a s y m^{2} \square}^{S O(m)} \\
& +\left(\chi_{V_{1}}+\chi_{V_{3}}+\cdots+\chi_{V_{r-2}}\right) \chi_{s y m^{2}}^{S O(2 N-r m)}+\chi_{\square}^{S O(m)} \chi_{\square}^{S O(2 N-r m)} \chi_{\frac{r-1}{2}}
\end{aligned}
$$

where $\operatorname{sym}^{2}\left(\right.$ asym $\left.^{2}\right)$ means the symmetric (asymmetric) square of representations. When $k+h^{\vee}=r$, the full character simplifies to

$$
P E\left[\frac{\left(q-q^{r}\right) \chi_{a d j}^{S O(2 N-r m)}}{(1-q)\left(1-q^{r}\right)}\right],
$$

which is the same as the vacuum character of the affine $\mathfrak{s o}_{2 N-r m}$ with level $\tilde{k}=-h^{\vee}+\frac{h^{\vee}}{r}$.

Verification of S duality conjecture: Consider a theory engineered by the following $(2,0)$ configuration

$$
\mathfrak{g}=\mathfrak{s l}_{3}, \quad \Phi=\frac{T_{3}}{z^{3}}+\frac{T_{2}}{z^{3}}+\frac{T_{1}}{z^{3}}, \quad f=\left[1^{3}\right] .
$$

Here $T_{i}$ are generic diagonal matrices. This theory has one exact marginal deformation, and the flavor symmetry is $U(1)^{2} \times S U(3)$. The weakly coupled gauge theory description is found in $[63,80]$ : the original theory is represented by a fourth punctured sphere with three identical $U(1)$ punctures and a $S U(3)$ puncture, while the weakly coupled gauge theory description is found by taking the degeneration limit of punctured sphere as shown in figure 2. The above $\mathrm{S}$ duality conjecture suggests that there is a symmetry exchanging three $U(1)$ punctures, and now we will use our index formula to confirm this speculation. The weakly coupled gauge theory is constructed by gauging the $S U(2)$ subgroup of a $D_{2}[S U(3)]$ theory 

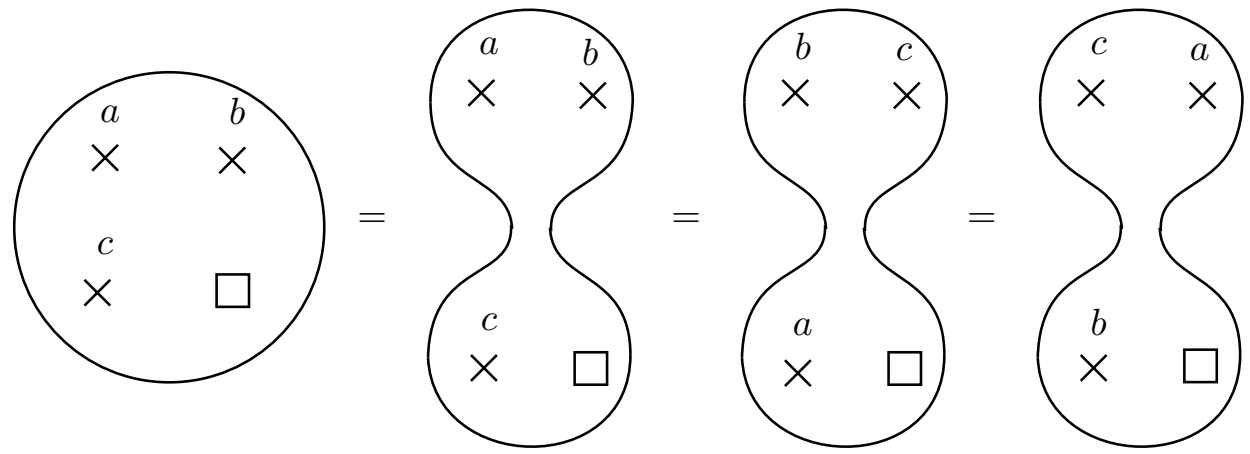

Figure 2: Three Duality frames of theory 4.35.

and a $D_{2}[S U(5)]$ theory. The $2 \mathrm{~d}$ VOA of $D_{2}[S U(3)]\left(D_{2}[S U(5)]\right)$ theory is $V^{-3 / 2}\left(\mathfrak{s l}_{3}\right)$ $\left(V^{-5 / 2}\left(\mathfrak{s l}_{5}\right)\right)$. The flavor symmetry of the gauged theory is $S U(3) \times U(1)_{a} \times U(1)_{b}$, and the Schur index is

$$
\mathcal{I}=\oint \frac{d z}{2 \pi i}\left(1-z^{2}\right)\left(1-\frac{1}{z^{2}}\right) \mathcal{I}^{D_{2}(S U(3))} \mathcal{I}^{V}(z ; q) \mathcal{I}^{D_{2}(S U(5))}
$$

with

$$
\mathcal{I}^{V}(z ; q)=(q ; q)^{2}\left(z^{2} q ; q\right)^{2}\left(z^{-2} q ; q\right)^{2}
$$

being the Schur index of the $S U(2)$ vector multiplet. Expanded in the power series of $q$, the index is

$$
\begin{aligned}
\mathcal{I}=1 & +q\left(2+\chi_{a d j}^{S U(3)}\right) \\
& +q^{2}\left[4+2 \chi_{a d j}^{S U(3)}+\chi_{s y m^{2} a d j}^{S U(3)}+\left(a+b+\frac{1}{a b}\right) \chi_{\square}^{S U(3)}+\left(\frac{1}{a}+\frac{1}{b}+a b\right) \chi_{\square}^{S U(3)}\right]+\cdots,
\end{aligned}
$$

where two $U(1)$ fugacities are defined as $a=e^{2 \pi \alpha\left(5 h_{1}+3 h_{2}\right)}$ and $b=e^{2 \pi \beta\left(5 h_{1}-3 h_{2}\right)}$ with $h_{1}$ and $h_{2}$ being Cartans of two remaining $U(1)$ 's in $S U(3)$ and $S U(5)$ after gauging the $S U(2)$. The index is not only symmetric under the exchange of $a$ and $b$, but also symmetric under the permutation of $a, b$ and $c$ if we replace $a b$ by $1 / c$. One can also see the same symmetry in higher order terms. This fact comes from the permutations symmetry of the three simple punctures in the $6 \mathrm{~d}$ construction shown in 2 . The third $U(1)$ is just formal because the coefficient of the term linear in $q$ indicates that there are only two $U(1)$ currents.

Let us now consider another theory which is engineered by following $(2,0)$ configuration

$$
\mathfrak{g}=\mathfrak{s l}_{4}, \quad \Phi=\frac{T_{3}}{z^{3}}+\frac{T_{2}}{z^{3}}+\frac{T_{1}}{z^{3}}, \quad f=[4] .
$$

Here $T_{i}$ are diagonal matrices. This theory has one exact marginal deformation and the flavor symmetry is $U(1)^{3}$. This theory is represented by an auxiliary fourth punctured sphere with four identical $U(1)$ punctures. The weakly coupled gauge theory description is found by taking the degeneration limit of this extra punctured sphere, see figure 3 and 

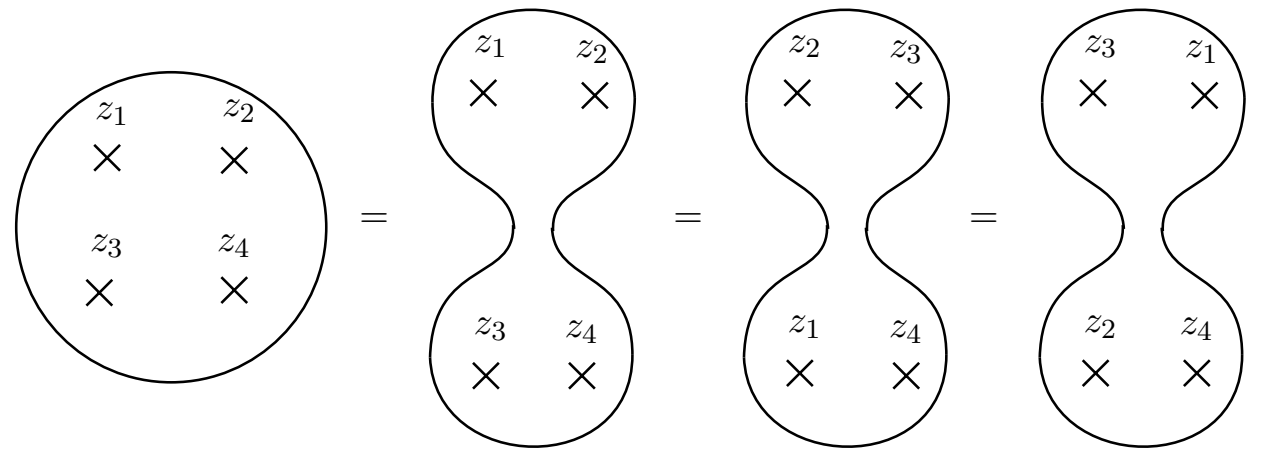

Figure 3: Duality frames of the theory constructed by gluing two $D_{2}[S U(3)]$ theory and one hypermultiplet.

also [81]. The above $\mathrm{S}$ duality picture suggests that there is a symmetry exchanging four $U(1)$ punctures, and we will use the index to confirm this conjecture. The weakly coupled gauge theory description is constructed by gauging the $S U(2)$ subgroup of two $D_{2}(S U(3))$ theories and a hypermultiplet which transforms as a fundamental of $S U(2)$ gauge group. The flavor symmetry of the gauged theory is $U(1)^{3}$, and the index is

$$
\mathcal{I}=1+3 q+\left(\sum_{i=1}^{4}\left(z_{i}+z_{i}^{-1}\right)\right) q^{\frac{3}{2}}+\left(10+\sum_{1 \leq i<j \leq 4} z_{i} z_{j}\right) q^{2}+\cdots
$$

with $z_{4}=\left(z_{1} z_{2} z_{3}\right)^{-1}$. Again, apart from the $S_{3}$ symmetry among three $U(1)$ 's, there is a hidden $S_{4}$ symmetry coming from the four simple punctures in the $6 \mathrm{~d}$ construction shown in 3. The fourth $U(1)$ is just formal because the terms linear in $q$ tells us that there are only three conserved currents.

\subsection{Explicit indices of $\left(A_{1}, G\right)$ theories}

Now consider the $\left(A_{1}, G\right)$ theory which can be engineered by the following three-fold singularity

$$
f_{A D E}(x, y, z)+w^{2}=0 .
$$

Here $f_{A D E}$ is a two dimensional $A D E$ singularity. The corresponding VOA for these theories are found in $[10,11,25,26,29]$ and summarized in table 5. The Schur index can 


\begin{tabular}{|c|c|c|c|}
\hline$\left(G, G^{\prime}\right)$ & $\left(J^{b}[k], f\right)$ & $\mathrm{VOA}$ & $f$ \\
\hline$\left(A_{1}, A_{2 N}\right)$ & $\left(A_{1}^{2}[2 N+1], f\right)$ & $\mathcal{W}^{-2+\frac{2}{2 N+3}}\left(A_{1}, f\right)$ & {$[2]$} \\
\hline$\left(A_{1}, A_{2 N-1}\right)$ & $\left(A_{N-1}^{N}[1], f\right)$ & $\mathcal{W}^{\frac{-N^{2}}{N+1}}\left(A_{N-1}, f\right)$ & {$[N-1,1]$} \\
\hline$\left(A_{1}, D_{2 N-1}\right)$ & $\left(A_{1}^{2}[2 N-3], f\right)$ & $\mathcal{W}^{-2+\frac{2}{2 N-1}}\left(A_{1}, f\right)$ & {$[1,1]$} \\
\hline$\left(A_{1}, D_{2 N-2}\right)$ & $\left(A_{N-1}^{N}[-1], f\right)$ & $\mathcal{W}^{\frac{-N^{2}+2 N}{N-1}}\left(A_{N-1}, f\right)$ & {$\left[N-2,1^{2}\right]$} \\
\hline$\left(A_{1}, E_{6}\right)$ & $\left(A_{2}^{3}[4], f\right)$ & $\mathcal{W}^{-\frac{18}{7}}\left(A_{2}, f\right)$ & {$[3]$} \\
\hline$\left(A_{1}, E_{7}\right)$ & $\left(A_{2}^{2}[3], f\right)$ & $\mathcal{W}^{-\frac{12}{5}}\left(A_{2}, f\right)$ & {$[2,1]$} \\
\hline$\left(A_{1}, E_{8}\right)$ & $\left(A_{2}^{3}[5], f\right)$ & $\mathcal{W}^{-\frac{21}{8}}\left(A_{2}, f\right)$ & {$[3]$} \\
\hline
\end{tabular}

Table 5: The VOA of $\left(A_{1}, A D E\right)$ theories.

be calculated by using our general index formula 4.19:

$$
\begin{aligned}
& \left(A_{1}, A_{2 N}\right): \quad \mathcal{I}=P E\left[\frac{q^{2}-q^{2 N+2}}{(1-q)\left(1-q^{2 N+3}\right)}\right], \\
& \left(A_{1}, A_{2 N-1}\right): \quad \mathcal{I}=P E\left[\frac{q+q^{2}+\left(a+a^{-1}\right) q^{\frac{N}{2}}-\left(a+a^{-1}\right) q^{\frac{N}{2}+2}-q^{N}-q^{N+1}}{(1-q)\left(1-q^{N+1}\right)}\right], \\
& \left(A_{1}, D_{2 N-1}\right): \quad \mathcal{I}=P E\left[\frac{\left(q-q^{2 N-1}\right) \chi_{1}(z)}{(1-q)\left(1-q^{2 N-1}\right)}\right], \\
& \left(A_{1}, D_{2 N-2}\right): \\
& \mathcal{I}=P E\left[\frac{\left(1+\chi_{1}(z)\right) q+\left(a+a^{-1}\right) \chi_{\frac{1}{2}}(z) q^{\frac{N-1}{2}}-\left(a+a^{-1}\right) \chi_{\frac{1}{2}}(z) q^{\frac{N+1}{2}}-\left(1+\chi_{1}(z)\right) q^{N-1}}{(1-q)\left(1-q^{N-1}\right)}\right], \\
& \left(A_{1}, E_{6}\right): \quad \mathcal{I}=P E\left[\frac{q^{2}+q^{3}-\left(q^{5}+q^{6}\right)}{(1-q)\left(1-q^{7}\right)}\right], \\
& \left(A_{1}, E_{7}\right): \quad \mathcal{I}=P E\left[\frac{q+\left(a+a^{-1}\right) q^{\frac{3}{2}}+q^{2}-q^{4}-\left(a+a^{-1}\right) q^{\frac{9}{2}}-q^{5}}{(1-q)\left(1-q^{5}\right)}\right], \\
& \left(A_{1}, E_{8}\right): \quad \mathcal{I}=P E\left[\frac{q^{2}+q^{3}-\left(q^{6}+q^{7}\right)}{(1-q)\left(1-q^{8}\right)}\right] .
\end{aligned}
$$

Here $a$ is the fugacity for $U(1)$ flavor symmetry and $z$ is the fugacity of $S U(2)$ flavor symmetry, and $\chi_{j}(z)$ is the character of the spin- $j$ representation of $S U(2)$.

\section{5 $\quad \tau \rightarrow 0$ limit and $a_{4 d}-c_{4 d}$}

The parameter $\tau$ is taking value on the upper half plane. As $\tau$ to zero, the character has the following asymptotic behavior [82]

$$
X_{V}(\tau) \sim \mathcal{A}(V) e^{\frac{\pi i \mathcal{G}(V)}{12 \tau}},
$$


where $A(V)$ is the amplitude and $G(V)$ is called the asymptotical growth. We have

$$
\mathcal{G}\left(L_{k}(\mathfrak{g})\right)=\left(1-\frac{h^{\vee}}{p q}\right) \operatorname{dim} \mathfrak{g}, \quad \mathcal{G}\left(W^{k}(\mathfrak{g}, f)\right)=\mathcal{G}\left(L_{k}(\mathfrak{g})\right)-\operatorname{dim} \mathfrak{f}
$$

for the level of AKM being $k=-h^{\vee}+\frac{p}{q}$. The dimension $\operatorname{dim} \mathfrak{f}$ is the dimension of the nilpotent orbit of $f$. On the other hand the above limit of the Schur index is studied in [83], and has the following asymptotic behavior

$$
\mathcal{I} \sim e^{-\frac{16 \pi^{2}}{3 \beta}\left(a_{4 d}-c_{4 d}\right)} \quad \text { as } \beta \rightarrow 0 .
$$

Therefore we have the identification $\beta=\frac{4 \pi}{3 i} \tau$, and

$$
a_{4 d}-c_{4 d}=-\frac{1}{48} \mathcal{G}
$$

where $\mathcal{G}$ is the growth defined before, see also [31] for the derivation of above formula. Furthermore, the Coulomb branch dictates another relation between $a_{4 d}$ and $c_{4 d}$

$$
2 a_{4 d}-c_{4 d}=\frac{1}{4} \sum\left(2 u_{i}-1\right) .
$$

with $u_{i}$ being the scaling dimension of Coulomb branch operator and the sum runs over all Coulomb branch operators. We can compute $a_{4 d}-c_{2 d}$ from above Coulomb branch formula, then use it to compare with the answer from index computed from $2 \mathrm{~d}$ VOA. This would provide a good check for our proposal of the $4 \mathrm{~d} / 2 \mathrm{~d}$ correspondence.

Example 1: Consider the case $f=$ regular, and take $\mathfrak{g}=A D E$, then the theory can also be engineered by a 3 -fold hypersurface singularity

$$
f_{A D E}(x, y, z)+w^{k}=0 .
$$

One can find its central charge $a_{4 d}$ and $c_{4 d}$ using the method presented in [77]. The computation is completely based on the Coulomb branch data, and results are

$$
a_{4 d}=\frac{l(k-1)\left(4 h^{\vee}(k+1)+4 k-1\right)}{48\left(h^{\vee}+k\right)}, \quad c_{4 d}=\frac{l(k-1)\left(h^{\vee}(k+1)+k\right)}{12\left(h^{\vee}+k\right)},
$$

where $l$ is the rank of $\mathfrak{g}$, and $h^{\vee}$ is the dual Coexeter number. Our VOA is $W^{k^{\prime}}\left(\mathfrak{g}, f_{\text {prin }}\right)$ algebra with $k^{\prime}=-h^{\vee}+\frac{h^{\vee}}{h^{\vee}+k}$, therefore (using 4.44)

$$
\mathcal{G}=\frac{l\left(h^{\vee}+k\right)-\operatorname{dim}(G)}{h^{\vee}+k}=\frac{l(k-1)}{h^{\vee}+k} .
$$

One can use the formula in 4.49 to check that $a_{4 d}-c_{4 d}$ is indeed $-\frac{1}{48} \mathcal{G}$.

Example 2: Consider the case $f=$ trivial, so that the $4 \mathrm{~d}$ SCFT has a $G$ flavor symmetry. The VOA is given by AKM $V^{k^{\prime}}(\mathfrak{g})$ with $k^{\prime}=-h^{\vee}+\frac{h^{\vee}}{h^{\vee}+k}$. The $4 \mathrm{~d}$ central charges $a_{4 d}$ and $c_{4 d}$ are

$$
a_{4 d}=\frac{\left(4 h^{\vee}+4 k-1\right)\left(h^{\vee}+k-1\right)}{48\left(h^{\vee}+k\right)} \operatorname{dim} \mathfrak{g}, \quad c_{4 d}=\frac{1}{12}\left(h^{\vee}+k-1\right) \operatorname{dim} \mathfrak{g} ;
$$

The growth (use 4.44) is

$$
\mathcal{G}=\frac{h^{\vee}+k-1}{h^{\vee}+k} \operatorname{dim} \mathfrak{g},
$$

which is again the same as $-48\left(a_{4 d}-c_{4 d}\right)$. 


\section{Zhu's $C_{2}$ algebra and the ring of the Schur sector}

Given a $2 \mathrm{~d}$ VOA $V$, one can associate an associative and commutative ring $R_{V}$ which is called Zhu's $C_{2}$ algebra. $R_{V}$ is in general an affine scheme and one can get a reduced affine ring which is further identified with the Higgs branch chiral ring of the corresponding $4 \mathrm{~d}$ theory. It is quite interesting to consider the reduced affine ring as one can learn the structure of Higgs branch chiral ring [29, 31, 66].

The new perspective of this paper states that the Zhu's $C_{2}$ algebra is actually more important than its reduced counterpart. On the one hand, if a 4d SCFT has no Higgs branch, the reduced affine ring is just trivial, however, the Zhu's $C_{2}$ algebra can still be quite non-trivial in this case. On the other hand, there are many 4d SCFT which shares the same Higgs branch so reduced affine rings of their associated VOAs are the same, however, their Zhu's $C_{2}$ algebras can still be very different, therefore the Zhu's $C_{2}$ algebra can be used to distinguish different $4 \mathrm{~d} \mathcal{N}=2$ SCFTs. Moreover, the Zhu's $C_{2}$ algebra can be thought as the classical limit of Zhu's algebra whose representation theory is closely related to the representation theory of the VOA, so the non-reduced version is definitely more important than its reduced version.

Since the reduced affine ring of Zhu's $C_{2}$ algebra gives the Higgs branch chiral ring, we might call Zhu's algebra as the ring of the Schur sector. The physical meaning of this ring seems quite interesting and we leave it for the future work. The main purpose of this section is to compute explicitly Zhu's $C_{2}$ algebras for VOAs considered in this paper.

\subsection{Zhu's $C_{2}$ algebra and Jacobi algebra}

Consider $W^{k^{\prime}}(\mathfrak{g}, f)$ algebra with $f$ being principle, so there is no flavor symmetry left. Recall $k^{\prime}=-h^{\vee}+\frac{h^{\vee}}{h^{\vee}+k}$. The corresponding $W$ algebra is just the minimal model of $W$-algebra. The character of the vacuum module takes the following form

$$
\operatorname{ch}_{W^{k^{\prime}}\left(g, f_{\text {prin }}\right)}(\tau)=P E\left[\frac{\sum_{i} q^{d_{i}}-q^{k-1}\left(\sum q^{d_{i}}\right)}{(1-q)\left(1-q^{h^{\vee}+k}\right)}\right], \quad k^{\prime}=-h^{\vee}+\frac{h^{\vee}}{h^{\vee}+k} .
$$

This $W$-algebra is strongly generated by a set of fields $W_{i}$ with scaling dimension $d_{i}$ which are just degrees of Casimirs of the Lie algebra $\mathfrak{g}$. These fields also generate the Zhu's algebra as the VOA is strongly finitely generated. From the character, one finds that there are $l$ singular vectors at levels $h^{\vee}+k-d_{i}, \quad i=1, \ldots, l$. To write down explicitly its Zhu's $C_{2}$ algebra, one need to analyze its singular vectors explicitly.

However we will take a different approach and discover a surprising appearance of the singularity theory and the Jacobi algebra. Since there is no flavor symmetry left, and it is believed that the Higgs branch is trivial, the Zhu's $C_{2}$ algebra is finite dimensional. Based on some concrete examples, we would like to conjecture that the Zhu's $C_{2}$ algebra of a minimal $W$-algebra is isomorphic to the Jacobi algebra of a quasi-homogeneous isolated singularity $F$.

Let us first review the associated Jacobi algebra of a quasi-homogeneous isolated singularity $F$. Consider a hypersurface singularity defined by a polynomial $F:\left(\mathbb{C}^{n}, 0\right) \rightarrow(\mathbb{C}, 0)$ with a $\mathbb{C}^{*}$ action

$$
F\left(\lambda^{\omega_{i}} z_{i}\right)=\lambda^{d} F(z)
$$


then one has the weight data associated with this singularity $F$ as $\left(w_{1}, \ldots, w_{n} ; d\right)$, and one might choose a particular normalization such that these weights are all integers (we do not require that they are pairwise co-prime though). The singularity is isolated if equations $F=\frac{\partial F}{\partial z_{i}}=0$ have a unique solution at $z_{i}=0$. The Jacobi algebra $J_{F}$ associated with $F$ is then defined as

$$
J_{F}=\mathbb{C}\left[z_{1}, \ldots, z_{n}\right] /\left\{\frac{\partial F}{\partial z_{1}}, \ldots, \frac{\partial F}{\partial z_{n}}\right\} .
$$

On the other hand, given a set of integral weights $\left(w_{1}, \ldots, w_{n} ; d\right)$, one might try to construct a polynomial $F$ such that $F$ has an isolated singularity at the origin. A necessary condition for this to happen is that for each variable $z_{i}$, there is at least one monomial of the following form

$$
\left\{z_{1} z_{i}^{a}, \ldots, z_{n} z_{i}^{a}\right\}
$$

The degree $d$ and weights $w_{i}$ provide a constraint on whether such monomial is possible or not.

Coming back to our problem of finding Zhu's $C_{2}$ algebras of $W$-algebra minimal models, we have already learned that the Zhu's algebra is generated by elements $W_{i}, i=1, \ldots, l$ with scaling dimension $d_{i}$. From the index, we have singular vectors at the level $h^{\vee}+k-d_{i}, \quad i=$ $1, \ldots, l$, and we conjecture that these singular vectors are enough to generate all relations, so the Zhu's algebra might take the following form

$$
\mathbb{C}\left[W_{1}, \ldots, W_{l}\right] /\left\{F_{1}, \ldots, F_{l}\right\} .
$$

Each $W_{i}$ has degree $d_{i}$ and $F_{i}$ has degree $h^{\vee}+k-d_{i}$. We would like now conjecture that the Zhu's algebra is isomorphic to a Jacobi algebra associated with a hypersurface singularity $F$ of type

$$
\left(d_{1}, \ldots, d_{l} ; h^{\vee}+k+1\right) .
$$

To find the explicit form of $F$, we simply write down the possible monomials from the set 5.4 for each variable $W_{i}$.

Example 1: Take $\mathfrak{g}=\mathfrak{s l}_{3}$ and $k=4$. The Zhu's algebra is generated by two variables $W_{2}, W_{3}$ with weights $(2,3 ; 8)$. The monomials in $F$ should be degree 8 , then for $W_{2}$ variable, we have a monomial $W_{2}^{4}$ which is in the set 5.4 , and for variable $W_{3}$, we have a monomial $W_{2} W_{3}^{2}$. So the polynomial $F=W_{2}^{4}+W_{2} W_{3}^{2}$, the Jacobi algebra is then

$$
\frac{\mathbb{C}\left[W_{2}, W_{3}\right]}{\left\{W_{2}^{3}+W_{3}^{2}, W_{2} W_{3}\right\}} \text {. }
$$

Example 2: For $\mathfrak{g}=\mathfrak{s l}_{n}$ and arbitrary $k$, we have the level-rank duality so that the same theory can be realized by $\mathfrak{g}=\mathfrak{s l}_{k}$ with the other data $n$. The associated Zhu's algebra of two descriptions should be equivalent. In this example, we show that this is indeed the case. Take $n=3, k=4$, and we have computed Zhu's algebra using the $\mathfrak{g}=\mathfrak{s l}_{3}$ description in the previous example. Using the $\mathfrak{s l}_{4}$ description, we have three generators $W_{2}, W_{3}, W_{4}$ with weights $(2,3,4 ; 8)$, and the degree $d$ of monomials in $F$ should be 8 . The polynomial is then $F^{\prime}=W_{2}^{4}+W_{2} W_{3}^{2}+W_{4}^{2}$, and it is well known that the Jacobi algebra of $F^{\prime}$ is the same as the Jacobi algebra associated with the polynomial $F=W_{2}^{4}+W_{2} W_{3}^{2}$. 


\subsection{Singular vector and general proposal for Zhu's $C_{2}$ algebra}

Once we know the singular vector of a VOA, we can actually compute the Zhu's $C_{2}$ algebra from the definition in section 2.1. Here we give some explicit examples.

Example 1: Consider the $\left(A_{1}, A_{2 N}\right)$ AD theory whose VOA is the $(2,2 N+3)$ minimal model of the Virosora algebra. This VOA is strongly generated by the energy-momentum tensor $T(z)$. The first non-trivial singular vector appears at level $2 N$ with the following form

$$
\left[\left(L_{-2}\right)^{N}+\ldots\right]|0\rangle
$$

where we ignore terms involving operators $L_{n}$ with $n<3$ as these terms give derivative fields, which are in the same class as [0] in the Zhu's $C_{2}$ algebra. In the VOA literature, the scaling dimension does not enter the expansion of fields, i.e. $a(z)=\sum_{n} a_{n} z^{-n-1}$, and one also use this convention for the stress tensor field $T(z)$. However, in physics literature, one usually use the convention $T(z)=\sum_{n} L_{n} z^{-n-2}$, so we have the identification $a_{n}=L_{n-1}$. Now in physics convention, the Verma module is generated by following vector

$$
L_{j_{1}} \ldots L_{j_{m}}|0\rangle, \quad j_{1} \leq \ldots j_{m} \leq 2
$$

The operator-states correspondence is

$$
L_{j_{1}} \ldots L_{j_{m}}|0\rangle, \rightarrow a_{j_{1} \ldots j_{m}}(z)=: \partial_{z}^{-j_{1}-2} T(z) \ldots \partial_{z}^{-j_{m}-2} T(z): .
$$

In particular $L_{-2}|0\rangle \rightarrow T(z)$, and $L_{-n}|0\rangle \rightarrow \partial^{n-2} T(z)$. If we expand the field $T(z)=$ $\sum_{n} a_{n} z^{-n-1}$, then $\left(\partial^{n-2} T(z)\right)_{-2}=a_{-n}=L_{-n-1}$. This implies that $L_{-n}$ with $n>3$ is the -2 modes of a vector in VOA. Now the $C_{1}$ subspace of our VOA (using the -1 shift in the mode expansion) is as

$$
C_{1}(V)=\left\{a_{-2} b \mid a, b \in V\right\} .
$$

So a state vector in 5.9 involves the raising operator $L_{-n}$ with $n<-3$ would be in $C_{1}(V)$ and is zero in the Zhu's $C_{2}$ algebra $V / C_{1}(V)$. The generator of Zhu's $C_{2}$ algebra is the state $L_{-2}|0\rangle \rightarrow T(z)$, and the singular vector 5.8 gives a relation

$$
T^{N}=0,
$$

where 0 means the fields involve derivatives. So Zhu's $C_{2}$ algebra is simply

$$
\mathbb{C}[T] / T^{N} .
$$

Let us now consider theory with $\mathfrak{g}=\mathfrak{s l}_{l+1}$, and the level $k^{\prime}=-\frac{l+1}{2}$, and $f$ is trivial. We take $l$ to be even, and this theory is also called $D_{2} S U(l+1)$. This theory has Coulomb branch spectrum $\left[\frac{3}{2}, \frac{5}{2}, \ldots, \frac{l+1}{2}\right]$, and flavor symmetry is $S U(l+1)$. The singular vector which generate the maximal proper ideal is found in [84]

$$
v=\left(\sum_{i=1}^{l} \frac{l-2 i+1}{l+1} h_{i}(-1) e_{\theta}(-1)-\sum_{i=1}^{l} e_{\epsilon_{1}-\epsilon_{i+1}}(-1) e_{\epsilon_{i+1}-\epsilon_{l+1}}(-1)-\frac{1}{2}(l-1) e_{\theta}(-2)\right)|0\rangle .
$$


Here $\theta$ is the longest root of Lie algebra $\mathfrak{s l}_{l+1}$ and $\theta=\alpha_{1}+\ldots+\alpha_{l}$ with $\alpha_{i}$ the set of simple roots. We use the convention that $\alpha_{i}=\epsilon_{i}-\epsilon_{i+1}$ are simple roots. So $\epsilon_{1}-\epsilon_{i+1}=\alpha_{1}+\ldots+\alpha_{i}$ and $\epsilon_{i+1}-\epsilon_{l+1}=\alpha_{i+1}+\ldots+\alpha_{l}$. The level of this singular vector is 2 , and it is the highest weight of the adjoint representation which matches the $-q^{2} \chi(z)$ term in the index formula $4.14\left(h^{\vee}+k\right.$ is 2 in this case).

Example 2: Consider $D_{2}[S U(3)]$ theory, and the corresponding VOA is $V^{-\frac{3}{2}}\left(\mathfrak{s l}_{3}\right)$. The VOA is strongly generated by the fields $x_{i} \in \mathfrak{s l}_{3}$, so the corresponding Zhu's $C_{2}$ algebra is also generated by $x_{i}$. We choose the basis of the Lie algebra to be $\left(h_{1}, h_{2}, e_{12}, e_{23}, e_{13}, e_{21}, e_{32}, e_{31}\right)$, where $h_{1}$ and $h_{2}$ generate the Cartan subalgebra, and $e_{i j}$ is the root vector of the root $\epsilon_{i}-\epsilon_{j}$. From our general index formula, we can see the image of the maximal proper ideal of $R_{V^{-\frac{3}{2}}\left(\mathfrak{s l}_{2}\right)}$ is an adjoint $\mathfrak{s l}_{3}$-module. We write down this ideal $I$ explicitly

$$
\begin{aligned}
\bar{v} & =\frac{1}{3}\left(h_{1}-h_{2}\right) e_{13}+e_{12} e_{23}, \\
\left(\operatorname{ad} e_{21}\right) \bar{v} & =-\frac{1}{3}\left(2 h_{1}+h_{2}\right) e_{23}+e_{21} e_{13}, \\
\left(\operatorname{ad} e_{32}\right) \bar{v} & =-\frac{1}{3}\left(h_{1}+2 h_{2}\right) e_{12}-e_{13} e_{32}, \\
\left(\operatorname{ad} e_{32}\right)\left(\operatorname{ad} e_{21}\right) \bar{v} & =-e_{12} e_{21}+e_{13} e_{31}+\frac{1}{3}\left(2 h_{1}+h_{2}\right) h_{2}, \\
\left(\operatorname{ad} e_{21}\right)\left(\operatorname{ad} e_{32}\right) \bar{v} & =-e_{23} e_{32}+e_{13} e_{31}+\frac{1}{3}\left(h_{1}+2 h_{2}\right) h_{1}, \\
\left(\operatorname{ad} e_{21}\right)\left(\operatorname{ad} e_{32}\right)\left(\operatorname{ad} e_{21}\right) \bar{v} & =\frac{1}{3}\left(h_{1}+2 h_{2}\right) e_{21}+e_{23} e_{31}, \\
\left(\operatorname{ad} e_{32}\right)\left(\operatorname{ad} e_{21}\right)\left(\operatorname{ad} e_{32}\right) \bar{v} & =\frac{1}{3}\left(2 h_{1}+h_{2}\right) e_{32}-e_{31} e_{12}, \\
\left(\operatorname{ad} e_{21}\right)\left(\operatorname{ad} e_{32}\right)\left(\operatorname{ad} e_{21}\right)\left(\operatorname{ad} e_{32}\right) \bar{v} & =\frac{1}{3}\left(h_{1}-h_{2}\right) e_{31}+e_{32} e_{21} .
\end{aligned}
$$

Here $h_{i}, i=1,2$ and $e_{i j}, i \neq j$ generate the polynomial ring $\mathbb{C}\left[h_{i}, e_{i j}\right]$ and then

$$
R_{V^{-\frac{3}{2}\left(\mathfrak{s l}_{2}\right)}}=\mathbb{C}\left[h_{i}, e_{i j}\right] / I .
$$

To get $X_{V^{-\frac{3}{2}\left(\mathfrak{s l}_{2}\right)}}$, one first work out the radical $I^{r}$ of $I$, which is

$$
\begin{aligned}
& \left(h_{1}-h_{2}\right) e_{13}+3 e_{12} e_{23}, \\
& \left(2 h_{1}+h_{2}\right) e_{23}-3 e_{21} e_{13}, \\
& \left(h_{1}+2 h_{2}\right) e_{12}+3 e_{13} e_{32}, \\
& h_{1}^{2}+4 e_{12} e_{21}+e_{13} e_{31}+e_{23} e_{32}, \\
& h_{1} h_{2}-2 e_{12} e_{21}+e_{13} e_{31}-2 e_{23} e_{32}, \\
& h_{2}^{2}+e_{12} e_{21}+e_{13} e_{31}+4 e_{23} e_{32}, \\
& \left(h_{1}+2 h_{2}\right) e_{21}+3 e_{23} e_{31}, \\
& \left(2 h_{1}+h_{2}\right) e_{32}-3 e_{31} e_{12}, \\
& \left(h_{1}-h_{2}\right) e_{31}+3 e_{32} e_{21},
\end{aligned}
$$


and $X_{V^{-\frac{3}{2}\left(\mathfrak{s l}_{2}\right)}}=\operatorname{spec}\left[\mathbb{C}\left[h_{i}, e_{i j}\right] / I^{r}\right]$. One can show that $X_{V^{-\frac{3}{2}\left(\mathfrak{s l}_{2}\right)}}$ is isomorphic to $\overline{\mathbb{O}}_{\text {min }}$ and has dimension 4. Moreover, the ideal $I^{r}$ has the same representation structure as the Joseph ideal of $\mathfrak{s l}_{3}$.

\subsection{A general proposal for Zhu's $C_{2}$ algebra}

Now, we would like to make a general conjecture of Zhu's $C_{2}$ algebras of our $W$-algebras $W^{k^{\prime}}(\mathfrak{g}, f)$. Recall that given a nilpotent element $f$ and its associated $\mathfrak{s l}_{2}$ triple, we have a subgroup $S U(2) \times G_{F}$ with $G_{F}$ the flavor symmetry group. The adjoint representation of $\mathfrak{g}$ is decomposed into representations of $S U(2) \times G_{F}$ as $a d j_{\mathfrak{g}} \rightarrow \oplus_{j}\left(V_{j} \otimes R_{j}\right)$. Given the structure of our simplified index formula, we would like to conjecture that the Zhu's $C_{2}$ algebra of our VOA has the following form:

1. Zhu's algebra is generated by the fields $W_{j}$ with scaling dimension $(1+j)$, and the number of such fields are given by $\operatorname{dim} R_{j}$, and they transform as $R_{j}$ representation of flavor group $G_{j}$.

2. Relations for fields $W_{j}$ 's are generated only by the set of singular vectors $v_{j}$ at level $q^{k+h^{\vee}-j}$ in the representation $R_{j}$.

3. The associated variety is simply $S_{f} \cap O_{k^{\prime}}$ [85]. Here $S_{f}$ is the Slodowy slice of $f$ and $O_{k^{\prime}}$ is the nilpotent orbit which is determined by the level $k^{\prime}$.

The above proposal is based on the structure of character formula presented in 4.19. The detailed form of the ideal is quite complicated, and we do not know a systematical way of writing down the ideal.

Here we give some conjectured form of Zhu's $C_{2}$ algebra for $\left(A_{1}, G\right)$ theories. Zhu's $C_{2}$ algebras for theories with no flavor symmetries can be decided using the method proposed in section 5.1.

$$
\begin{array}{ll}
\left(A_{1}, A_{2 N}\right): & R_{V}=J_{I}, \quad I=T^{N+2}, \\
\left(A_{1}, E_{6}\right): & R_{V}=J_{I}, \quad I=T^{4}+T W^{2}, \\
\left(A_{1}, E_{8}\right): & R_{V}=J_{I}, \quad I=W^{3}+T W^{3} .
\end{array}
$$

Here $R_{V}$ is the Zhu's $C_{2}$ algebra and $J_{I}$ is the associated Jacobi algebra of polynomial $I$. $T$ has scaling dimension two, and $W$ has scaling dimension three.

Zhu's $C_{2}$ algebras for theories with one $U(1)$ flavor symmetry are computed as follows (for $\left(A_{1}, A_{2 N-1}\right)$ theories)

$$
\begin{aligned}
& \left(A_{1}, A_{2 N-1}\right): \mathbb{C}\left[J, T, W_{+}, W_{-}\right] / I, \\
& \text { with } I=\left\{W_{+}\left(J^{2}+T\right), W_{-}\left(J^{2}+T\right), W_{+} W_{-}+\sum_{i=0}^{N-2 i \geq 0} J^{N-2 i} T^{i}, W_{+} W_{-} J+\sum_{i=0}^{N-2 i \geq 0} J^{N+1-2 i} T^{i}\right\},
\end{aligned}
$$


where $J$ has scaling dimension one. $T$ has scaling dimension two, and $W_{ \pm}$have scaling dimension $\frac{N}{2}$ and $U(1)$ charge \pm 1 . And for $\left(A_{1}, E_{7}\right)$ theory, we have:

$$
\begin{aligned}
& \left(A_{1}, E_{7}\right): \quad R_{V}=\mathbb{C}\left[J, W_{+}, W_{-}, T\right] / I, \\
& \text { with } \\
& I=\left\{J^{4}+J^{2} T+T^{2}+J W_{+} W_{-}, W_{+}\left(J^{3}+J T+W_{+} W_{-}\right),\right. \\
& \left.\quad W_{-}\left(J^{3}+J T+W_{+} W_{-}\right), J^{5}+J^{3} T+J T^{2}+W_{+} W_{-}\left(J^{2}+T\right)\right\},
\end{aligned}
$$

here $J$ has scaling dimension one, and $W$ has scaling dimension two, and $W_{ \pm}$has scaling dimension $\frac{3}{2}$.

And the Zhu's $C_{2}$ algebras for theories with at least one $S U(2)(U(2))$ flavor symmetry are computed as follows

$$
\begin{aligned}
& \left(A_{1}, D_{2 N-2}\right): \quad R_{V}=\mathbb{C}\left[J_{j}^{i}, W_{i}^{+}, W_{i}^{-}\right] / I \\
& \text { with } I=\left\{\left(\delta_{i}^{j} \operatorname{tr} J+J_{i}^{j}\right) W_{j}^{+},\left(\delta_{i}^{j} \operatorname{tr} J+J_{i}^{j}\right) W_{j}^{-}, W_{i}^{+} W_{j}^{-}+\left(\sum_{l=0}^{N}\left(\operatorname{tr} J^{l}\right)\left(J^{N-l}\right)_{i}^{k}\right) \epsilon_{k j}\right\} .
\end{aligned}
$$

Here dimension one operators $J_{j}^{i}$,s are in the adjoint representation of $U(2)$ with $i, j=1,2$. Dimension $\frac{n-1}{2}$ operators $W_{i}^{ \pm}$form fundamental and antifundamental representations of $U(2)$ respectively. In those cases, the ideal is found by following strategy: a): since we know the grading of each polynomial $f_{i}$ in the ideal, we first write all possible combinations of monomials in $f_{i}$; b): we also know the Higgs branch dimension in each case, i.e. $n_{h}=1$ for $\left(A_{1}, A_{2 N-1}\right)$ and $\left(A_{1}, E_{7}\right)$ case, and $n_{h}=2$ for $\left(A_{1}, D_{2 N-2}\right)$ case. So a consistent condition is that the dimension of the reduced ring should be equal to the dimension of the Higgs branch.

\section{Kazhdan filtration and Macdonald index}

The Schur index of a four dimensional $\mathcal{N}=2$ SCFT has only one fugacity $q$, and it is identified with the character of the vacuum module of its associated $2 \mathrm{~d}$ VOA. The Macdonald index also counts Schur operators, but with two fugacities $q$ and $T$, see section 2. Since the $4 \mathrm{~d} / 2 \mathrm{~d}$ correspondence is actually between the Schur sector and the VOA itself, it should be possible to recover the Macdonald index from the structure of VOA. The VOA has one natural grading which is just the eigenvalue of zero mode of $2 \mathrm{~d}$ energy-momentum tensor $T(z)$. To recover the Macdonald index, one need to find another grading. Such grading is found for some AD theories in [22]. Here we generalize their results to all $W$-algebras $W^{k^{\prime}}(\mathfrak{g}, f)$ considered in this paper, and a crucial ingredient is a new type of filtration called Kazhdan filtration defined for our W algebra.

First let us consider the universal affine VOA $V^{k}(\mathfrak{g})$ associated with an AKM algebra. For a Lie algebra $\mathfrak{g}$, one has its universal enveloping algebra $\mathcal{U}(\mathfrak{g})$ [86]. By the PBW theorem, $V^{k}(\mathfrak{g})$ has a PBW basis consisting of monomials of the form:

$$
x_{n_{1}}^{i_{1}} \ldots x_{n_{m}}^{i_{m}}|0\rangle .
$$


where $n_{1} \leq n_{2} \ldots \leq n_{m}<0$, and if $n_{j}=n_{j+1}$, then $i_{j} \leq i_{j+1}$. Here $x^{i}$ is an ordered basis of Lie algebra $\mathfrak{g}$. The universal affine VOA $V^{k}(\mathfrak{g})$ is then isomorphic to its universal enveloping algebra $\mathcal{U}(\mathfrak{g})$. The PBW filtration on the VOA is then defined as follows

$$
K_{-1} \mathcal{U}(g)=0, \quad K_{0} \mathcal{U}(g)=\mathbb{C}, \quad K_{p} \mathcal{U}(g)=\mathfrak{g} F_{p-1} \mathcal{U}(g)+F_{p-1} \mathcal{U}(g) .
$$

Here $F_{p} \mathcal{U}(g)$ is the PBW filtration on $\mathcal{U}(g)$. The VOA is generated by $\operatorname{dim}(\mathfrak{g})$ fields $x_{i}(z)$ and all states are spanned by derivatives of these generating fields. The PBW filtration at level $p$ simply includes those states with at most $p$ generating fields $x^{i}(z)$ (the number of derivatives on those fields is not limited though), i.e., one assign $\mathrm{T}$ grading one to the fundamental fields $x^{i}(z)$. Since each $x_{i}(z)$ gives a $\hat{\mathcal{B}}_{1}$ type operators, its $T$ grading is just one, and it is natural to identify PBW grading as the one giving $T$ grading. This is almost right, but there is an important subtly that we will discuss later.

Now consider $W$-algebra $W^{k}(\mathfrak{g}, f)$. Given an $\mathfrak{s l}_{2}$ triple $(x, e, f)$, the Lie algebra has the following decomposition

$$
\mathfrak{g}=\oplus_{j \in \mathbb{Z} / 2} \mathfrak{g}_{j} .
$$

For each element $v_{j}$ in $\mathfrak{g}_{j}^{f}$, one has a generator $J_{v_{j}}$ in VOA with scaling dimension $1+j$ (for $j \geq 0$ ). Now set $F_{p} \mathcal{U}(\mathfrak{g})[j]=\left\{[x, u]=j u, u \in \mathcal{U}_{p}(\mathfrak{g})\right\}$, one then has the following (grading by half-integer) Kazhdan filtration

$$
K_{p} \mathcal{U}(\mathfrak{g})=\sum_{i+j \leq p} F_{i} \mathcal{U}(\mathfrak{g})[j]
$$

In particular, the generator $J_{v_{j}} \in \mathfrak{g}_{j}^{f}$ is in $F_{1} \mathcal{U}(\mathfrak{g})$ and $j$ value is just $j$, so it is in space $K_{s} \mathcal{U}(\mathfrak{g})$ with $s \geq j+1$.

We need to modify a little bit on above Kazhdan filtration so that this grading can give us Macdonald index. Since our VOA is strongly generated by the fields $J_{v_{j}}$, we can assign a grading to VOA by using the grading of the generators from Kazhdan filtration. The rule is following: since $J_{v_{0}}$ gives $\hat{\mathcal{B}}_{1}$ type operator, we assign $T$ grading one to it. For the other fields $J_{v_{j}}$ though, we have to assign grading $j$ to it (from Kazhdan grading). Using this modified grading, we now have an increasing filtration on our VOA (which we still call Kazhdan filtration):

$$
K_{0} \subset K_{1} \subset K_{2} \subset \ldots
$$

and a decreasing Li's filtration (see section 2)

$$
F_{0} \supset F_{1} \supset F_{2} \supset \ldots
$$

and we can form the following double graded space

$$
H^{p, k}=\frac{F^{p} \cap K_{k}}{F^{p+1} \cap K_{k}}+K_{k-1} .
$$

And each vector in subspace $H^{p, k}$ has two gradings: one grading is just the Kazhdan grading $k$, and other one is the conformal grading $\Delta$ (notice that $\Delta$ is different from $p$ ). Using above double grading, we define Macdonald index as follows:

$$
\mathcal{I}(q, T)=\sum_{H^{p, k}} q^{\Delta-\frac{c}{24}} T^{k}
$$


In the above consideration, the Kazhdan filtration is defined by choosing a generating set. In particular, in the AKM case, we choose $x^{i}(z)$ (with $x^{i}$ generating Lie algebra $\mathfrak{g}$ ) as the generating set. Now according to Sugawara construction, the energy momentum tensor $T(z) \sim x(z)^{2}$. If we use the Kazhdan filtration with $x^{i}(z)$ as generating set, $x^{2}$ would be in $K_{2}$ and has $T$ grading two which is inconsistent with the fact that the $T$ grading of $T(z)$ field should be just one. To remedy this situation, we use a strategy following [22]: we add $T(z)$ to our generating set and assign $T$ grading one to it, and imposing the relation $T(z) \sim x(z)^{2}$. Now VOA is strongly generated by the fields $x^{i}(z)$ and $T(z)$, and we have a similar Kazhdan filtration using the grading of generating fields. Now $T(z)$ contributes $q^{2} T$ to the index, however, the relation $T(z)-x(z)^{2}=0$ is actually in space $K_{2}$ and actually contributes $-q^{2} T^{2}$ to the index.

Example: Consider $\left(A_{1}, D_{2 N-1}\right)$ theory. The corresponding VOA is $V_{-2+\frac{2}{2 N-1}}(\mathfrak{s l}(2))$, and it has a singular vector at level $2 N-1$ transforming in adjoint representation, which actually contributes to index a term $-q^{2 N-1} T^{N} \chi_{a d j}(z)$. The Macdonald index then has the following form

$$
\mathcal{I}(q, T)=P E\left[\frac{q T \chi_{a d j}(z)+q^{2} T-q^{2} T^{2}-q^{2 N-1} T^{N} \chi_{a d j}(z)}{(1-q)\left(1-q^{2 N-1}\right)}+f(q, T, z)\right] .
$$

Here $f(q, 1, z)=0$ so that we recover Schur index in $T=1$ limit. In the above computation, Zhu's $C_{2}$ algebra actually plays a crucial role. Let us take $N=2$ for an example. We use a generating set $(a, b, c, T)$ for our VOA so that the Zhu's $C_{2}$ algebra is also generated by these fields. The Zhu's $C_{2}$ algebra is

$$
\frac{C[a, b, c, T]}{\left\{T-\left(a b+c^{2}\right), a T, b T, c T\right\}} .
$$

where the ideal has four generators. The first one contributes to the index with a term $-q^{2} T^{2}$, and the last three contribute with a term $-q^{3} T^{2} \chi_{a d j}(z)$.

We do not have the closed formula for the Macdonald index for the general case, but we do know first few terms (in the case of AKM, one need to add the contribution from energy momentum tensor.)

$$
\mathcal{I}(q, T)=P E\left[\frac{q \sum q^{j} T^{j+\left[\frac{h^{\vee}-j}{h^{\vee}}\right]} \chi_{R_{j}}(z)-(q T)^{h^{\vee}+k} \sum q^{-j} T^{-\left[\frac{h^{\vee}+k-j}{h^{\vee}}\right]-j} \chi_{R_{j}}(z)}{(1-q)}+\ldots\right]
$$

Here $R_{j}$ is the representation of flavor symmetry group $G_{F}$, and $[a]$ means that we take the integral part of the number $a$ inside the square bracket.

\section{Conclusion}

It is quite difficult to understand the Schur sector of a general $\mathcal{N}=2 \mathrm{SCFT}$ due to the fact that most of these theories are strongly coupled, and no powerful tools such as the Seiberg-Witten geometry of the Coulomb branch is available. However, the correspondence between $4 \mathrm{~d} \mathcal{N}=2$ SCFTs and $2 \mathrm{~d}$ VOAs makes understanding of the Schur sector possible 
when the corresponding $2 \mathrm{~d} \mathrm{VOA}$ is known. In the previous work [56], we have identified the associated $2 \mathrm{~d}$ VOAs for a large class of $4 \mathrm{~d} \mathcal{N}=2$ SCFTs constructed from $6 \mathrm{~d}(2,0)$ theories. In this paper, we use the knowledge of $2 \mathrm{~d}$ VOA to learn lots of interesting properties of the Schur sector:

- The Schur index is computed from the vacuum character of $W$-algebra and can be put in a surprisingly simple form.

- The associated Zhu's $C_{2}$ algebra can be computed from the VOA and can be regarded as the ring associated with the Schur sector.

- One can use the Kazhdan filtration of the $W$-algebra to compute the Macdonald index.

The Zhu's $C_{2}$ algebra can be thought as the associated ring of the Schur sector. This algebra is in general quite complicated, and for some subset of theories, we found a surprising isomorphism between the Zhu's $C_{2}$ algebra and the Jacobi algebra of a hypersurface singularity. It would be interesting to further check our proposal. It would be also interesting to further study the physical meaning of this algebra. Moreover, one can have an associative (but not commutative) algebra which is called Zhu's algebra. This algebra controls lots of interesting information of the representation theory of the VOA, and it would be interesting to understand the physical meaning of Zhu's algebra as well.

We now would like to make some speculations on generators of Schur sector ring of models considered in this paper. The generator has the following contribution to Macdonald index $q^{1+j} T^{j}, j>0$ and $q T$ for $j=0$. Given a Schur operator $\hat{\mathcal{C}}_{R,\left(j_{1}, j_{2}\right)}$, its contribution to Macdonald index is $q^{2+R+j_{1}+j_{2}} T^{1+R+j_{2}-j_{1}}$ (see 2.2). Since there are three independent quantum numbers for a Schur operator and the Macdonald index can only capture two quantum numbers, we can not completely determine the Schur operator type corresponding to the generators. However, based on some known examples. we make the following conjecture: all generators are just scalars $\hat{\mathcal{B}}_{R+1}=\hat{\mathcal{C}}_{R,(-1 / 2,-1 / 2)}$ and $\hat{\mathcal{C}}_{R,(0,0)}$. It would be interesting to verify this conjecture.

One can construct new $\mathcal{N}=2$ SCFTs by gauging $\mathrm{AD}$ theories (They are called $\mathrm{AD}$ matters) considered in this paper, and the corresponding VOA of the gauged theory can be found from the cosets of $W$-algebras associated with AD matter [56]. The Schur index of the gauged system can also be computed using the index of the $\mathrm{AD}$ matters. We have used this strategy to check $\mathrm{S}$ duality conjecture proposed in $[39,57,58]$. Now one could have a different $\mathrm{S}$ duality where new $\mathrm{AD}$ matter would appear, and these $\mathrm{AD}$ matter is not included in theories considered in this paper. However, using the index of the full theory found from the original $\mathrm{S}$ duality frame and known results of other components in this new duality frame, it is possible to find indices of these new AD matters. Using the above method, it might be possible to get Schur indices of all AD theories constructed from $6 \mathrm{~d}$ $(2,0)$ theories.

In this paper, we mainly focus on the vacuum module of $W$-algebra. Other modules also play important roles in the study of four dimensional $\mathcal{N}=2$ SCFTs [24, 25, 37]. These modules will be studied in a forthcoming paper [87]. 


\section{Acknowledgements}

Authors would like to thank Peng Shan for helpful discussions. DX and WY are supported by Yau mathematical sciences center at Tsinghua University. WY is also supported by the young overseas high-level talents introduction plan.

\section{A Notation of special functions}

The convention of special functions are summarized in this section. First the definition of the $\mathrm{q}$-Pochhammer symbol $(x ; q)$ is

$$
(x ; q)=\prod_{i=0}^{\infty}\left(1-x q^{i}\right),
$$

and $(x ; q)_{i}$ is defined as

$$
(x ; q)_{i}=\frac{(x ; q)}{\left(x q^{i} ; q\right)} .
$$

The definition of the plethystic exponential is

$$
P E[f(a, \cdots)]=e^{\sum_{n=1}^{\infty} \frac{1}{n} f\left(a^{n}, \cdots\right)},
$$

therefore

$$
P E[x]=e^{\sum_{n=1}^{\infty} \frac{1}{n} x^{n}}=\frac{1}{1-x}
$$

and

$$
P E\left[\frac{x}{1-q}\right]=e^{\sum_{n=1}^{\infty} \frac{1}{n} \frac{x^{n}}{1-q^{n}}}=\prod_{i=0}^{\infty} \frac{1}{1-x q^{i}}=\frac{1}{(x ; q)} .
$$

The $\eta$-function $\eta(\tau)$ is defined as

$$
\eta(\tau)=q^{\frac{1}{24}} \prod_{n=1}^{\infty}\left(1-q^{n}\right)=q^{\frac{1}{24}}(q ; q),
$$

with $q=e^{2 \pi i \tau}$. And four Jacobian theta functions are defined as

$$
\begin{aligned}
& \theta_{00}(z, \tau)=q^{-\frac{1}{24}} \eta(\tau) \prod_{n=1}^{\infty}\left(1+e^{2 \pi i z} q^{n-\frac{1}{2}}\right)\left(1+e^{-2 \pi i z} q^{n-\frac{1}{2}}\right), \\
& \theta_{01}(z, \tau)=q^{-\frac{1}{24}} \eta(\tau) \prod_{n=1}^{\infty}\left(1-e^{2 \pi i z} q^{n-\frac{1}{2}}\right)\left(1-e^{-2 \pi i z} q^{n-\frac{1}{2}}\right) \\
& \theta_{10}(z, \tau)=q^{\frac{1}{12}} e^{-\pi i z} \eta(\tau) \prod_{n=1}^{\infty}\left(1+e^{-2 \pi i z} q^{n}\right)\left(1+e^{2 \pi i z} q^{n-1}\right) \\
& \theta_{11}(z, \tau)=-i q^{\frac{1}{12}} e^{-\pi i z} \eta(\tau) \prod_{n=1}^{\infty}\left(1-e^{-2 \pi i z} q^{n}\right)\left(1-e^{2 \pi i z} q^{n-1}\right) .
\end{aligned}
$$

One can also rewrite all theta functions in terms of q-Pochhammers or plethystic exponentials, which plays an important role in the main text. 


\section{References}

[1] F. Dolan and H. Osborn, On short and semi-short representations for four-dimensional superconformal symmetry, Annals Phys. 307 (2003) 41 [hep-th/0209056].

[2] P. C. Argyres, Y. Lu and M. Martone, Seiberg-Witten geometries for Coulomb branch chiral rings which are not freely generated, JHEP 06 (2017) 144 [1704.05110].

[3] A. Gadde, L. Rastelli, S. S. Razamat and W. Yan, The 4d Superconformal Index from q-deformed 2d Yang-Mills, Phys.Rev.Lett. 106 (2011) 241602 [1104.3850].

[4] A. Gadde, L. Rastelli, S. S. Razamat and W. Yan, Gauge Theories and Macdonald Polynomials, Commun.Math.Phys. 319 (2013) 147 [1110.3740].

[5] C. Beem, M. Lemos, P. Liendo, W. Peelaers, L. Rastelli and B. C. van Rees, Infinite Chiral Symmetry in Four Dimensions, Commun. Math. Phys. 336 (2015) 1359 [1312.5344].

[6] C. Beem, W. Peelaers, L. Rastelli and B. C. van Rees, Chiral algebras of class S, JHEP 1505 (2015) 020 [1408.6522].

[7] M. Lemos and W. Peelaers, Chiral Algebras for Trinion Theories, JHEP 02 (2015) 113 [1411.3252].

[8] M. Buican and T. Nishinaka, Argyres-Douglas theories, $S^{1}$ reductions, and topological symmetries, J. Phys. A49 (2016) 045401 [1505.06205].

[9] C. Cordova and S.-H. Shao, Schur Indices, BPS Particles, and Argyres-Douglas Theories, JHEP 01 (2016) 040 [1506.00265].

[10] M. Buican and T. Nishinaka, Argyres-Douglas Theories, the Macdonald Index, and an RG Inequality, JHEP 02 (2016) 159 [1509.05402].

[11] J. Song, Superconformal indices of generalized Argyres-Douglas theories from 2d TQFT, JHEP 02 (2016) 045 [1509.06730].

[12] M. Lemos and P. Liendo, Bootstrapping $\mathcal{N}=2$ chiral correlators, 1510.03866.

[13] S. Cecotti, J. Song, C. Vafa and W. Yan, Superconformal Index, BPS Monodromy and Chiral Algebras, JHEP 11 (2017) 013 [1511.01516].

[14] M. Lemos and P. Liendo, $\mathcal{N}=2$ central charge bounds from $2 d$ chiral algebras, JHEP 04 (2016) 004 [1511.07449].

[15] T. Nishinaka and Y. Tachikawa, On 4 d rank-one $\mathcal{N}=3$ superconformal field theories, JHEP 09 (2016) 116 [1602.01503].

[16] M. Buican and T. Nishinaka, Conformal Manifolds in Four Dimensions and Chiral Algebras, J. Phys. A49 (2016) 465401 [1603.00887].

[17] D. Xie, W. Yan and S.-T. Yau, Chiral algebra of Argyres-Douglas theory from M5 brane, 1604.02155.

[18] C. Cordova, D. Gaiotto and S.-H. Shao, Infrared Computations of Defect Schur Indices, JHEP 11 (2016) 106 [1606.08429].

[19] T. Arakawa and K. Kawasetsu, Quasi-lisse vertex algebras and modular linear differential equations, 1610.05865.

[20] M. Lemos, P. Liendo, C. Meneghelli and V. Mitev, Bootstrapping $\mathcal{N}=3$ superconformal theories, JHEP 04 (2017) 032 [1612.01536]. 
[21] F. Bonetti and L. Rastelli, Supersymmetric localization in $A d S_{5}$ and the protected chiral algebra, JHEP 08 (2018) 098 [1612.06514].

[22] J. Song, Macdonald Index and Chiral Algebra, JHEP 08 (2017) 044 [1612.08956].

[23] T. Creutzig, W-algebras for Argyres-Douglas theories, 1701.05926.

[24] L. Fredrickson, D. Pei, W. Yan and K. Ye, Argyres-Douglas Theories, Chiral Algebras and Wild Hitchin Characters, 1701.08782.

[25] C. Cordova, D. Gaiotto and S.-H. Shao, Surface Defect Indices and 2d-4d BPS States, 1703.02525.

[26] C. Cordova, D. Gaiotto and S.-H. Shao, Surface Defects and Chiral Algebras, JHEP 05 (2017) 140 [1704.01955].

[27] M. Dedushenko, S. Gukov and P. Putrov, Vertex algebras and 4-manifold invariants, 1705.01645.

[28] M. Buican and T. Nishinaka, On Irregular Singularity Wave Functions and Superconformal Indices, JHEP 09 (2017) 066 [1705.07173].

[29] J. Song, D. Xie and W. Yan, Vertex operator algebras of Argyres-Douglas theories from M5-branes, JHEP 12 (2017) 123 [1706.01607].

[30] M. Buican, Z. Laczko and T. Nishinaka, $\mathcal{N}=2$ S-duality revisited, JHEP 09 (2017) 087 [1706.03797].

[31] C. Beem and L. Rastelli, Vertex operator algebras, Higgs branches, and modular differential equations, JHEP 08 (2018) 114 [1707.07679].

[32] Y. Pan and W. Peelaers, Chiral Algebras, Localization and Surface Defects, JHEP 02 (2018) 138 [1710.04306].

[33] M. Fluder and J. Song, Four-dimensional Lens Space Index from Two-dimensional Chiral Algebra, JHEP 07 (2018) 073 [1710.06029].

[34] M. Buican and Z. Laczko, Nonunitary Lagrangians and unitary non-Lagrangian conformal field theories, Phys. Rev. Lett. 120 (2018) 081601 [1711.09949].

[35] J. Choi and T. Nishinaka, On the chiral algebra of Argyres-Douglas theories and S-duality, JHEP 04 (2018) 004 [1711.07941].

[36] T. Arakawa, Representation theory of $W$-algebras and Higgs branch conjecture, in International Congress of Mathematicians (ICM 2018) Rio de Janeiro, Brazil, August 1-9, 2018, 2017, 1712.07331.

[37] C. Kozçaz, S. Shakirov and W. Yan, Argyres-Douglas Theories, Modularity of Minimal Models and Refined Chern-Simons, 1801.08316.

[38] K. Costello and D. Gaiotto, Vertex Operator Algebras and $3 d \mathcal{N}=4$ gauge theories, 1804.06460 .

[39] Y. Wang and D. Xie, Codimension-two defects and Argyres-Douglas theories from outer-automorphism twist in $6 d(2,0)$ theories, 1805.08839.

[40] B. Feigin and S. Gukov, VOA $\left[M_{4}\right], 1806.02470$.

[41] V. Niarchos, Geometry of Higgs-branch superconformal primary bundles, Phys. Rev. D98 (2018) 065012 [1807.04296]. 
[42] T. Creutzig, Logarithmic W-algebras and Argyres-Douglas theories at higher rank, JHEP 11 (2018) 188 [1809.01725].

[43] M. Dedushenko, S. Gukov, H. Nakajima, D. Pei and K. Ye, $3 d$ TQFTs from Argyres-Douglas theories, 1809.04638.

[44] F. Bonetti, C. Meneghelli and L. Rastelli, VOAs labelled by complex reflection groups and $4 d$ SCFTs, 1810.03612.

[45] T. Arakawa, Chiral algebras of class $\mathcal{S}$ and Moore-Tachikawa symplectic varieties, 1811.01577.

[46] K. Costello, T. Creutzig and D. Gaiotto, Higgs and Coulomb branches from vertex operator algebras, 1811.03958.

[47] T. Nishinaka, S. Sasa and R.-D. Zhu, On the Correspondence between Surface Operators in Argyres-Douglas Theories and Modules of Chiral Algebra, 1811.11772.

[48] P. Agarwal, S. Lee and J. Song, Vanishing OPE Coefficients in $4 d N=2$ SCFTs, 1812.04743.

[49] C. Beem, Flavor symmetries and unitarity bounds in $\mathcal{N}=2$ SCFTs, 1812.06099.

[50] K. Kiyoshige and T. Nishinaka, OPE Selection Rules for Schur Multiplets in $4 D \mathcal{N}=2$ Superconformal Field Theories, 1812.06394.

[51] M. Buican and Z. Laczko, Rationalizing CFTs and Anyonic Imprints on Higgs Branches, 1901.07591.

[52] Y. Pan and W. Peelaers, Schur correlation functions on $S^{3} \times S^{1}, 1903.03623$.

[53] C. Beem, C. Meneghelli and L. Rastelli, Free Field Realizations from the Higgs Branch, 1903.07624.

[54] J. Oh and J. Yagi, Chiral algebras from $\Omega$-deformation, 1903.11123.

[55] M. Dedushenko and M. Fluder, Chiral Algebra, Localization, Modularity, Surface defects, And All That, 1904.02704.

[56] D. Xie and W. Yan, $W$ algebra, Cosets and VOAs for $4 d \mathcal{N}=2$ SCFT from M5 branes, 1902.02838.

[57] D. Xie, General Argyres-Douglas Theory, JHEP 1301 (2013) 100 [1204.2270].

[58] Y. Wang and D. Xie, Classification of Argyres-Douglas theories from M5 branes, 1509.00847.

[59] P. Bouwknegt and K. Schoutens, W symmetry in conformal field theory, Physics Reports 223 (1993) 183.

[60] V. Kac, S.-S. Roan and M. Wakimoto, Quantum reduction for affine superalgebras, Communications in mathematical physics 241 (2003) 307.

[61] T. Arakawa, Introduction to w-algebras and their representation theory, in Perspectives in Lie Theory, pp. 179-250, Springer, (2017).

[62] V. G. Kac and M. Wakimoto, A remark on boundary level admissible representations, Comptes Rendus Mathematique 355 (2017) 128.

[63] D. Xie and S.-T. Yau, Argyres-Douglas matter and N=2 dualities, 1701.01123.

[64] D. Xie and K. Ye, Argyres-Douglas matter and S-duality: Part II, JHEP 03 (2018) 186 [1711.06684]. 
[65] Y. Zhu, Modular invariance of characters of vertex operator algebras, Journal of the American Mathematical Society 9 (1996) 237.

[66] T. Arakawa, Chiral algebras of class s and moore-tachikawa symplectic varieties, arXiv preprint arXiv:1811.01577 (2018) .

[67] T. Arakawa, Rationality of w-algebras: Principal nilpotent cases, Annals of Mathematics $\mathbf{1 8 2}$ (2012) .

[68] M. Buican, T. Nishinaka and C. Papageorgakis, Constraints on chiral operators in $\mathcal{N}=2$ SCFTs, JHEP 12 (2014) 095 [1407.2835].

[69] V. G. Kac, Vertex algebras for beginners, no. 10. American Mathematical Soc., 1998.

[70] H. Li, Abelianizing vertex algebras, Communications in Mathematical Physics 259 (2005) 391.

[71] T. Arakawa and K. Kawasetsu, Quasi-lisse vertex algebras and modular linear differential equations, 1610.05865.

[72] P. Liendo, I. Ramirez and J. Seo, Stress-tensor OPE in $\mathcal{N}=2$ superconformal theories, JHEP 02 (2016) 019 [1509.00033].

[73] D. Xie, $\mathcal{N}=2$ SCFT with minimal flavor central charge, 1712.03244.

[74] D. Gaiotto, N=2 dualities, JHEP 1208 (2012) 034 [0904.2715].

[75] D. Gaiotto, G. W. Moore and A. Neitzke, Wall-crossing, Hitchin Systems, and the WKB Approximation, 0907.3987.

[76] M. Reeder, P. Levy, J.-K. Yu and B. H. Gross, Gradings of positive rank on simple lie algebras, Transformation Groups 17 (2012) 1123.

[77] D. Xie and S.-T. Yau, 4d N=2 SCFT and singularity theory Part I: Classification, 1510.01324.

[78] O. Chacaltana, J. Distler and Y. Tachikawa, Nilpotent orbits and codimension-two defects of 6d $N=(2,0)$ theories, Int. J. Mod. Phys. A28 (2013) 1340006 [1203.2930].

[79] T. Arakawa and A. Moreau, Singularities of nilpotent slodowy slices and collapsing levels for $w$-algebras, in preparation .

[80] D. Xie and S.-T. Yau, New $N=2$ dualities, 1602.03529.

[81] M. Buican, S. Giacomelli, T. Nishinaka and C. Papageorgakis, Argyres-Douglas Theories and S-Duality, JHEP 1502 (2015) 185 [1411.6026].

[82] V. G. Kac and M. Wakimoto, Classification of modular invariant representations of affine algebras. in Infinite Dimensional Lie Algebras and Groups, Adv. Ser. Math. Phys. 7 (1989) 138 .

[83] L. Di Pietro and Z. Komargodski, Cardy formulae for SUSY theories in $d=4$ and $d=6$, JHEP 12 (2014) 031 [1407.6061].

[84] O. Perse, Vertex operator algebras associated to certain admissible modules for affine lie algebras of type a, Glasnik Matematicki 43 (2007) 41 [0707.4129].

[85] T. Arakawa, Associated varieties of modules over Kac-Moody algebras and $C_{2}$-cofiniteness of W-algebras, Int. Math. Res. Not. IMRN (2015) 11605.

[86] J. E. Humphreys, Introduction to Lie Algebra and Representation Theory. 1972. 
[87] P. Shan, D. Xie and W. Yan, in preparation, . 